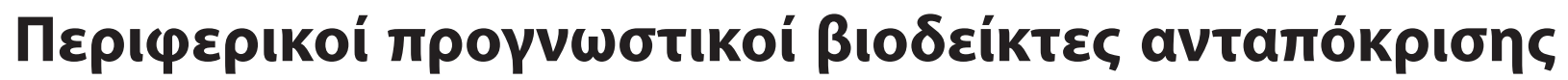

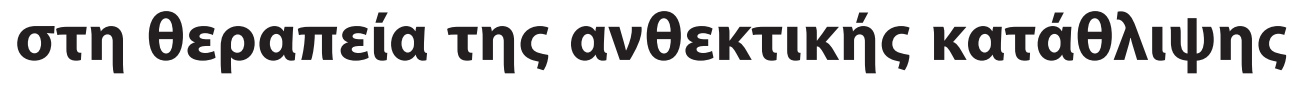

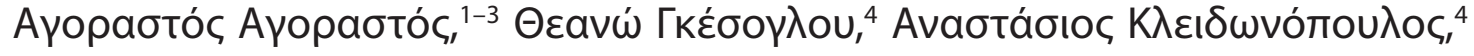

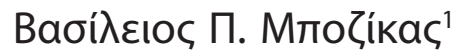

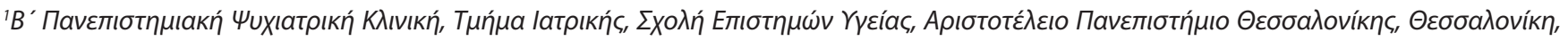 \\ ${ }^{2}$ VA Center of Excellence for Stress and Mental Health (CESAMH), VA San Diego Healthcare System, La Jolla, San Diego, CA, USA,

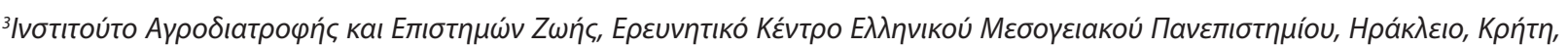

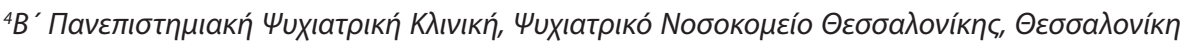

\section{ПЕРIАНЧН}

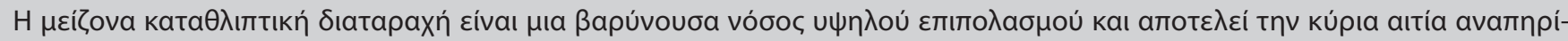

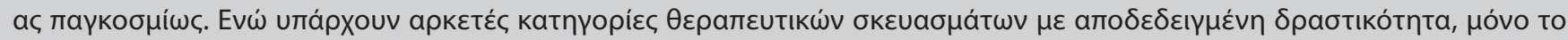

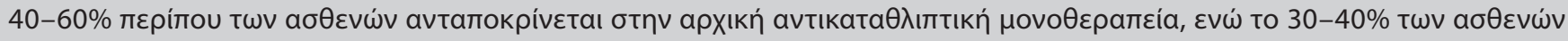

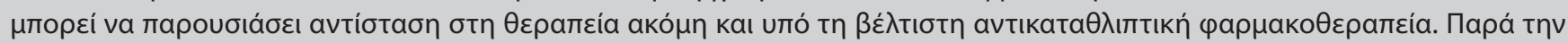

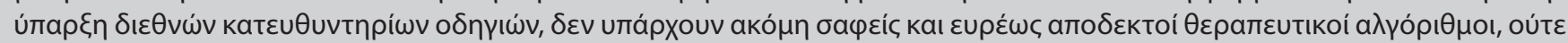

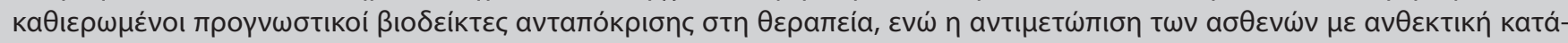

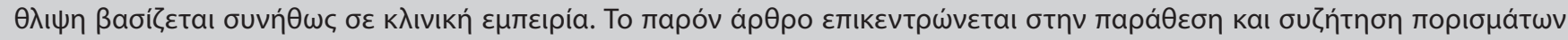

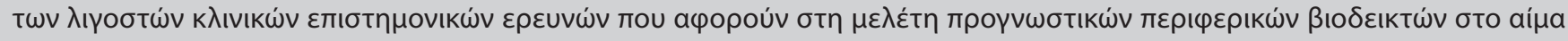

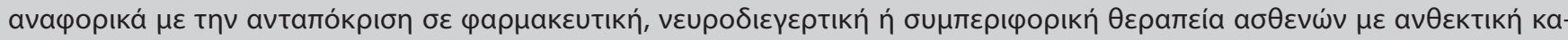

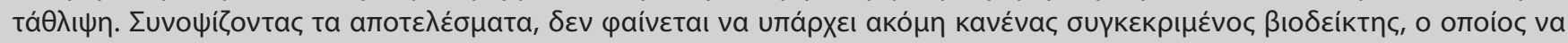

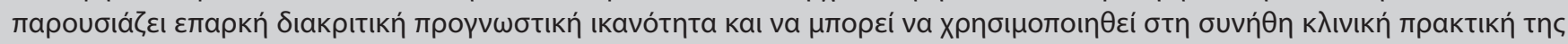

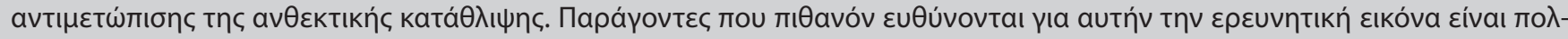

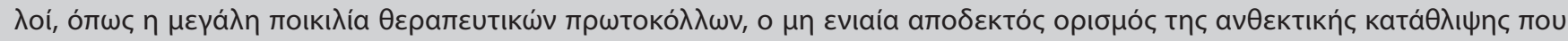

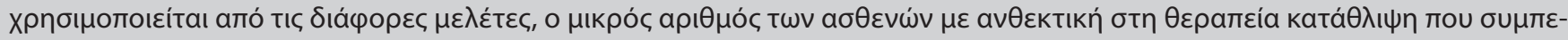

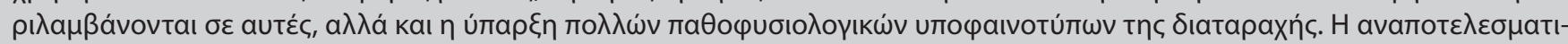

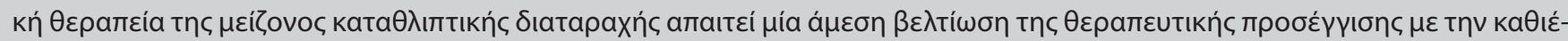

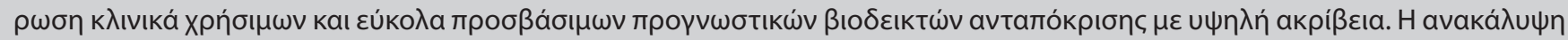

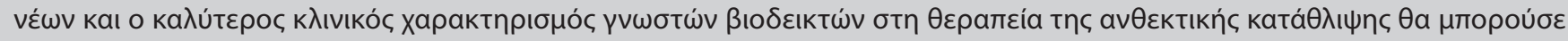

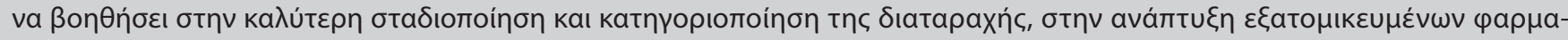

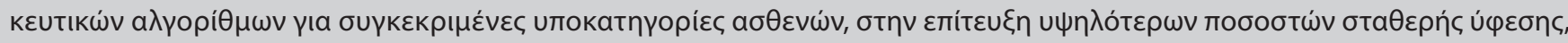

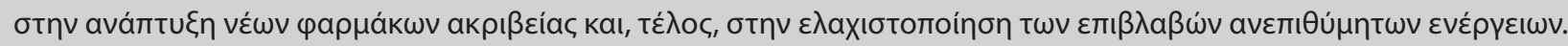

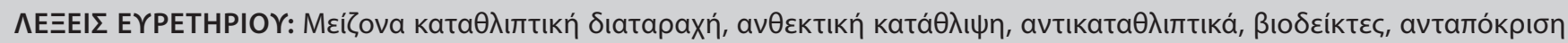

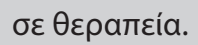

\section{Eıoapwyń}

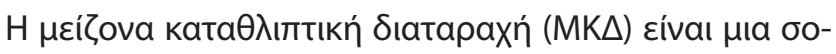

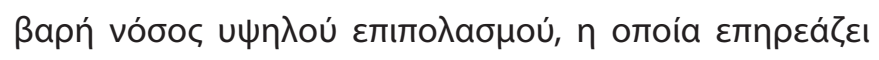

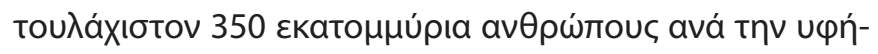

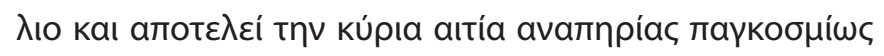

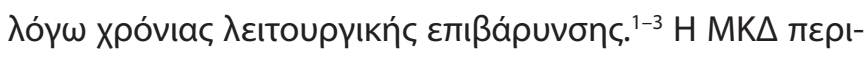

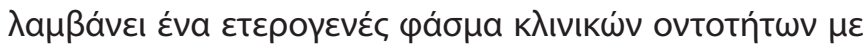

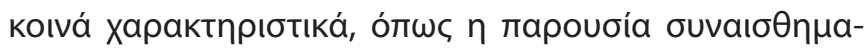

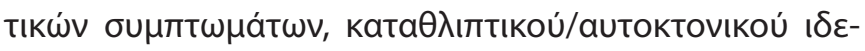

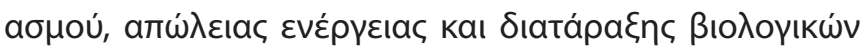

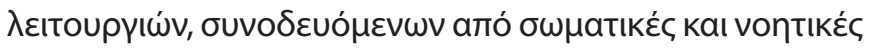




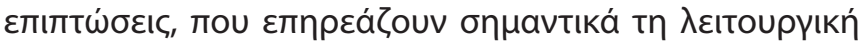

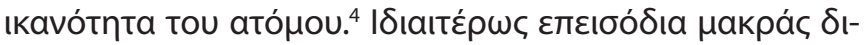

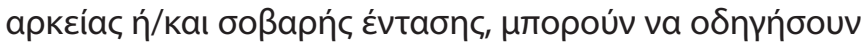

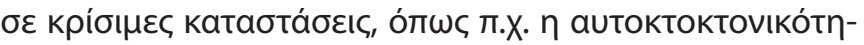

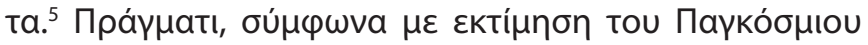

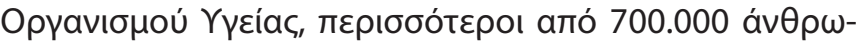

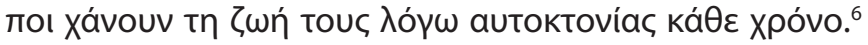

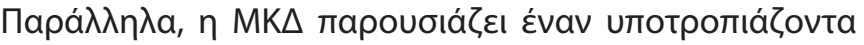

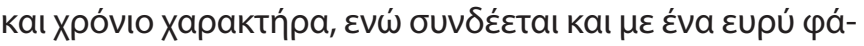

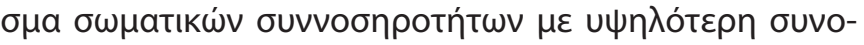

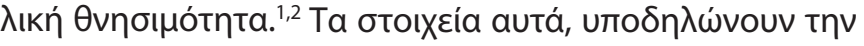

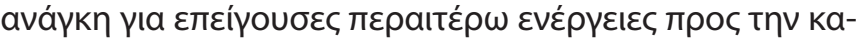

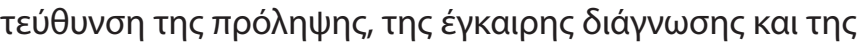

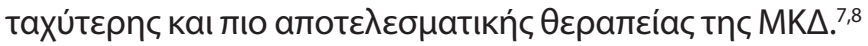

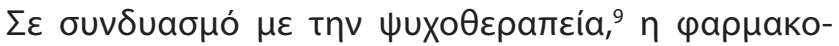

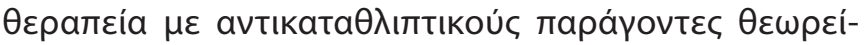

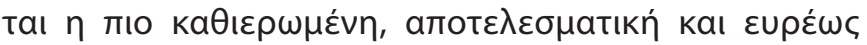

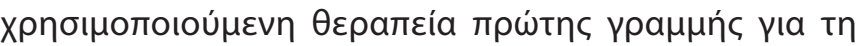

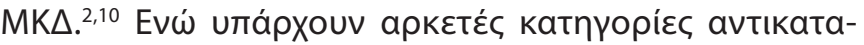

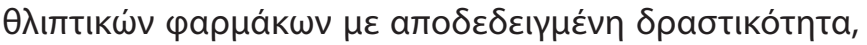

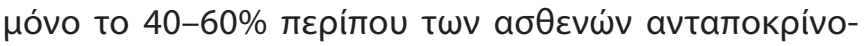

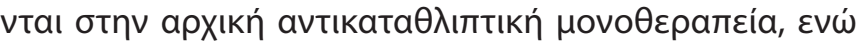

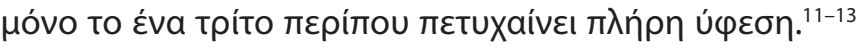

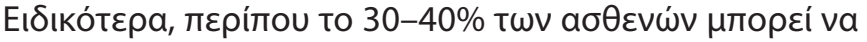

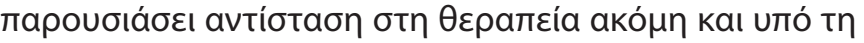

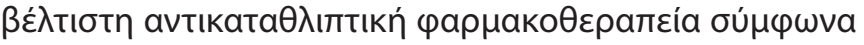

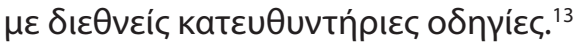

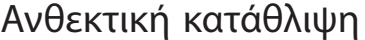

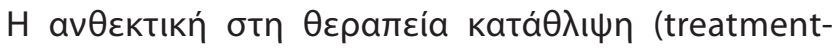
resistant depression, TRD) паратпрвітаı пврі́тои бто

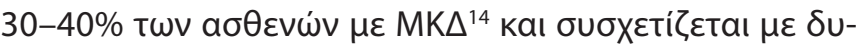

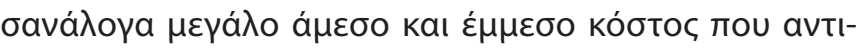

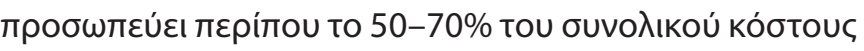

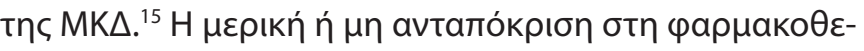

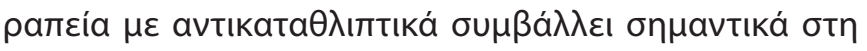

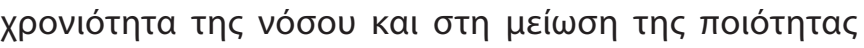

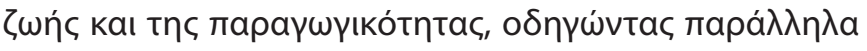

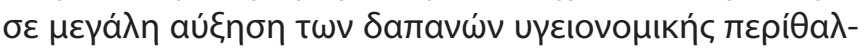

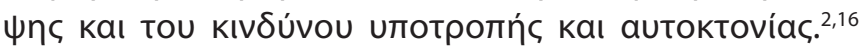

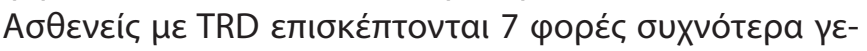

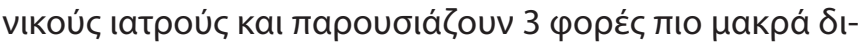

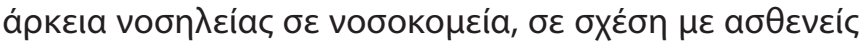

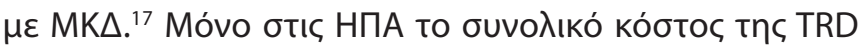

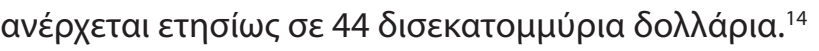

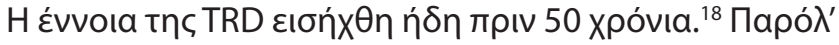

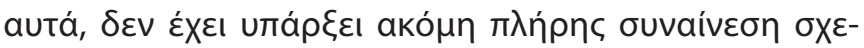

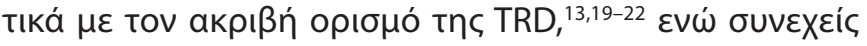

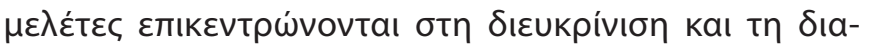

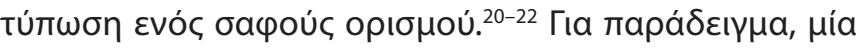

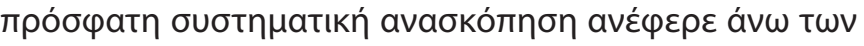

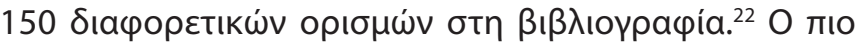

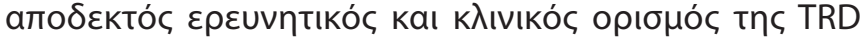

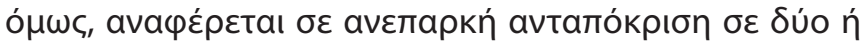

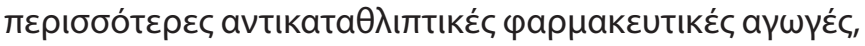

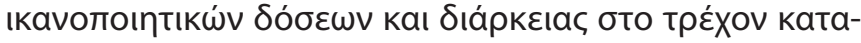

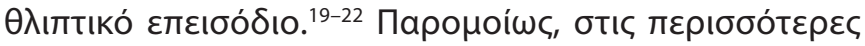

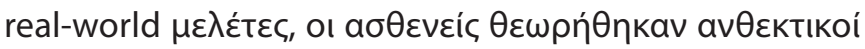

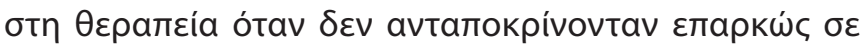

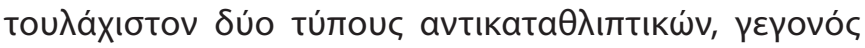

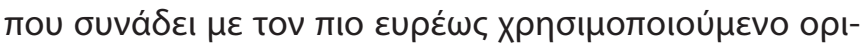

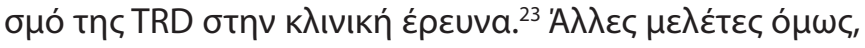

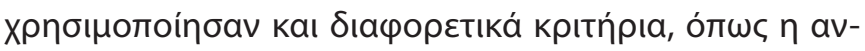

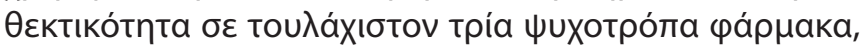

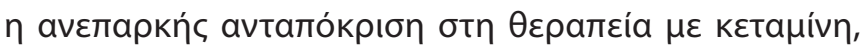

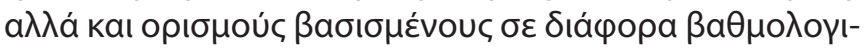

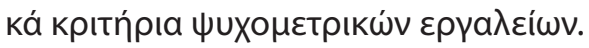

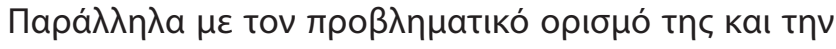

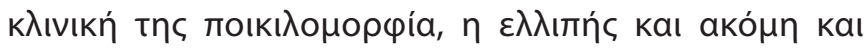

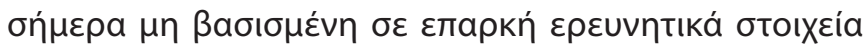

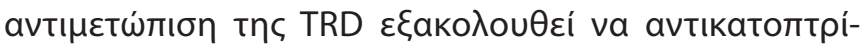

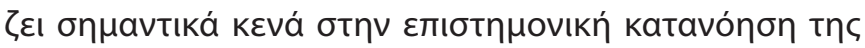

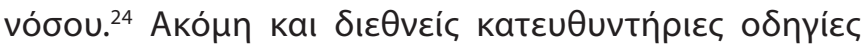

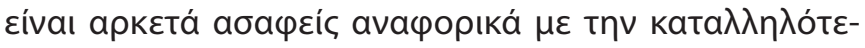

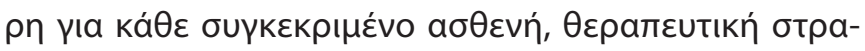

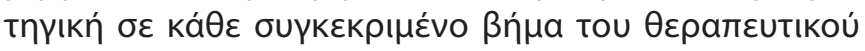

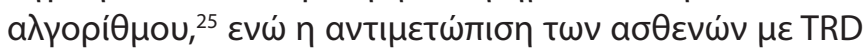

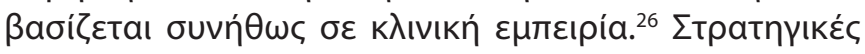

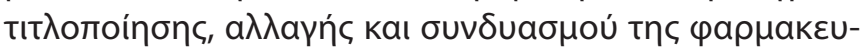

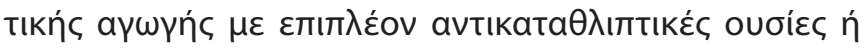

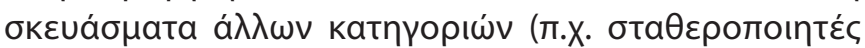

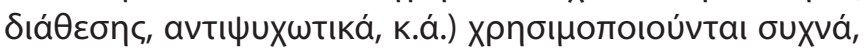

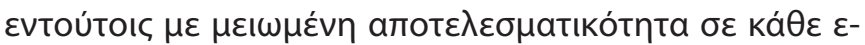

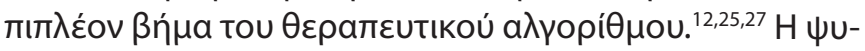

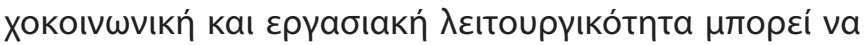

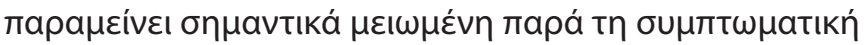

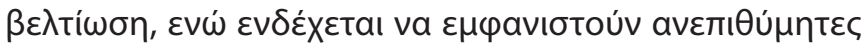

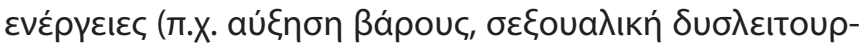

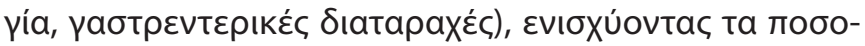

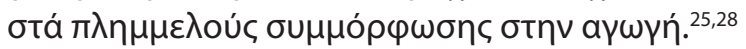

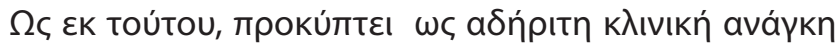

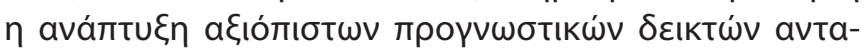

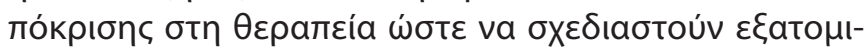

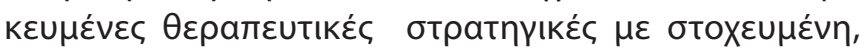

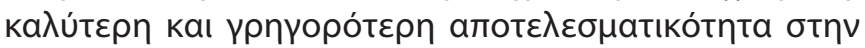

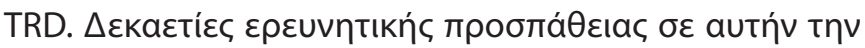

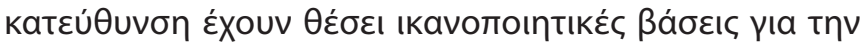

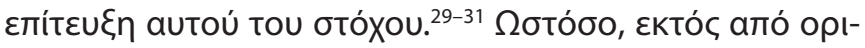

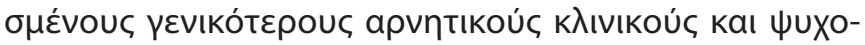

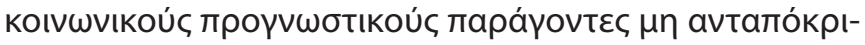

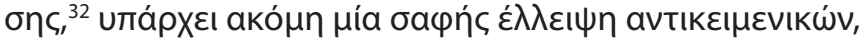

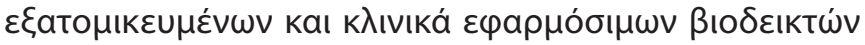

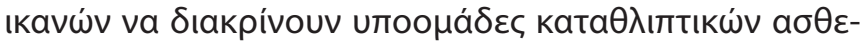

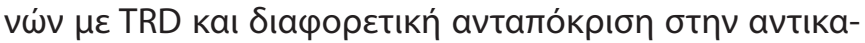

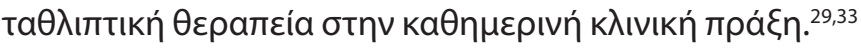




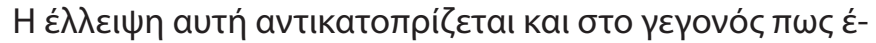

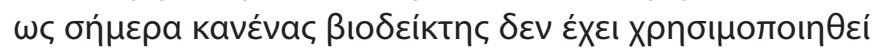

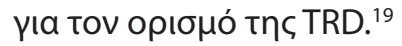

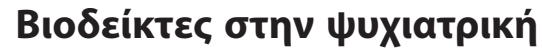

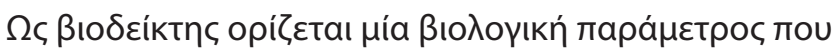

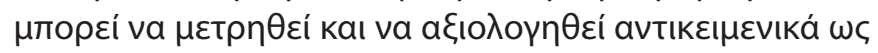

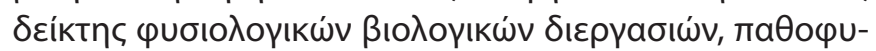

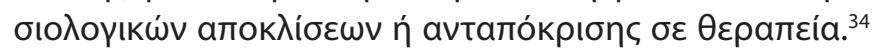

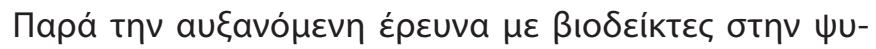

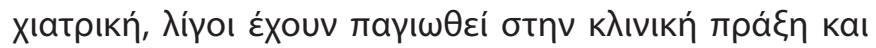

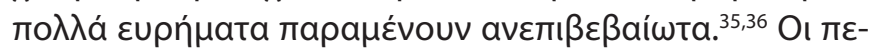

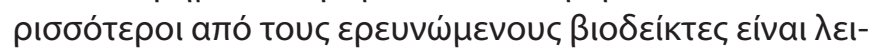

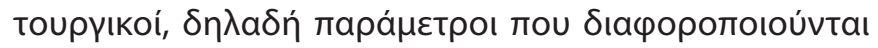

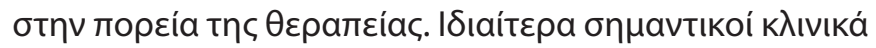

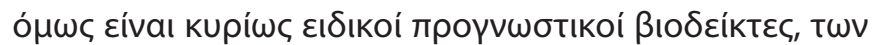

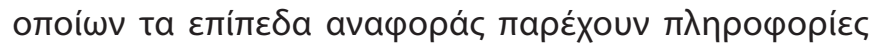

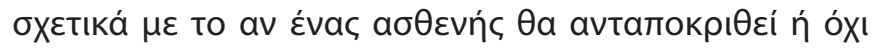

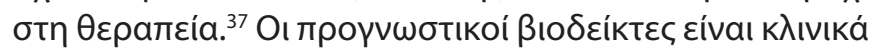

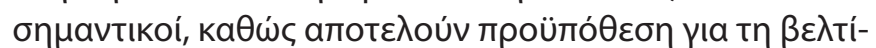

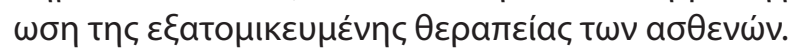

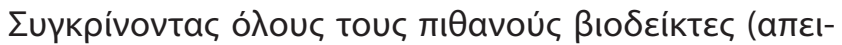

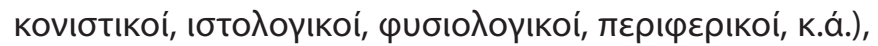

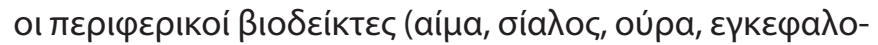

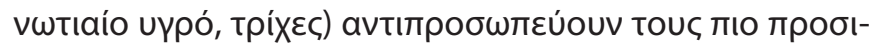

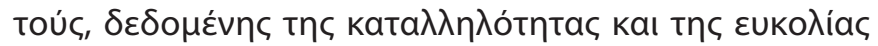

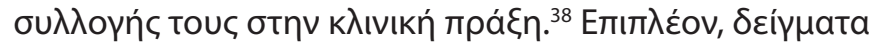

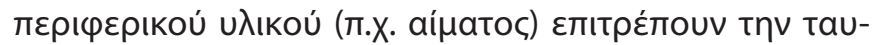

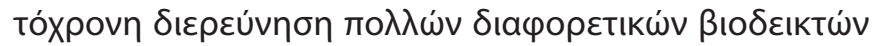

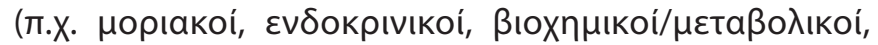

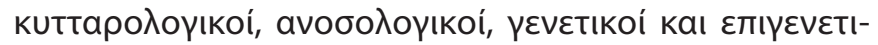

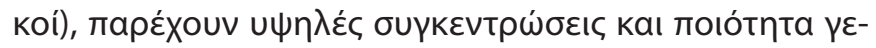

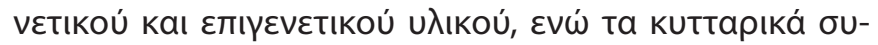

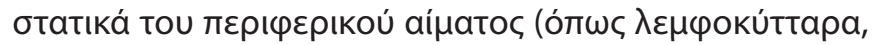

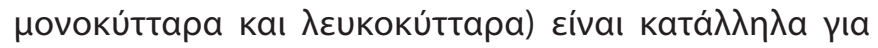

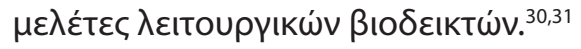

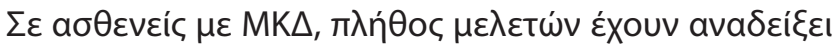

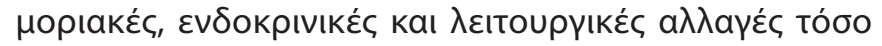

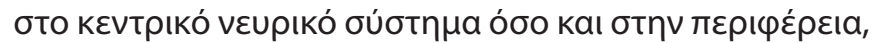

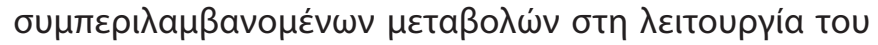

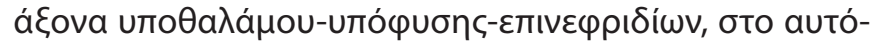

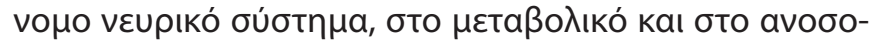

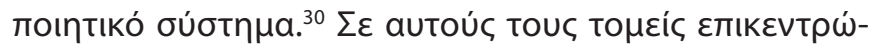

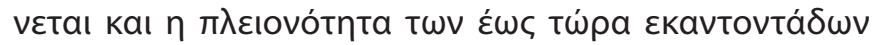

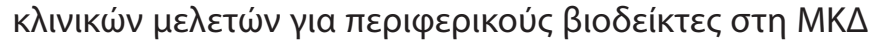

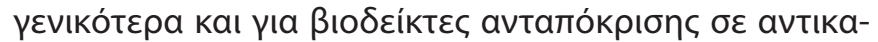

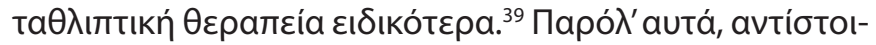

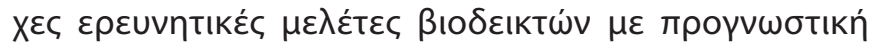

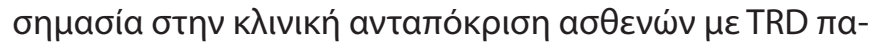

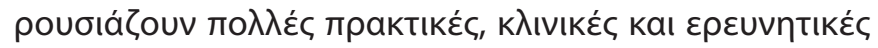

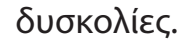

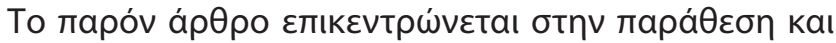

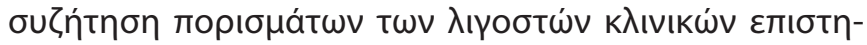

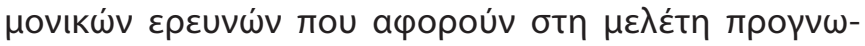

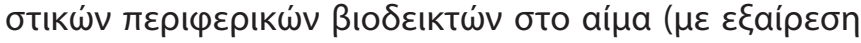

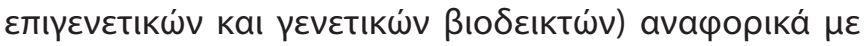

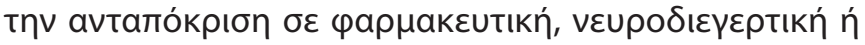

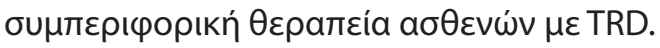

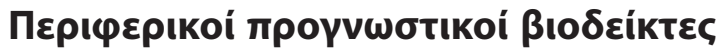

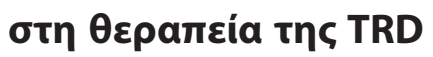

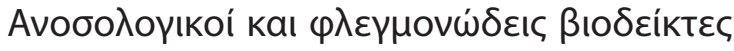

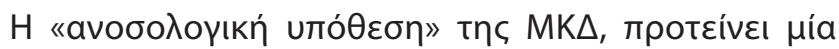

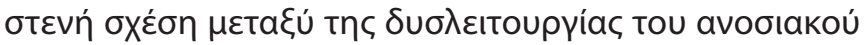

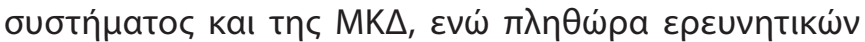

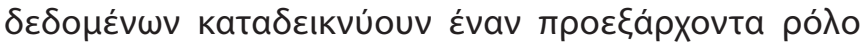

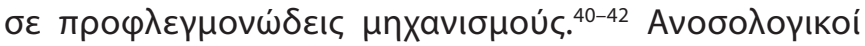

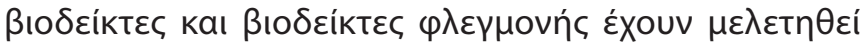

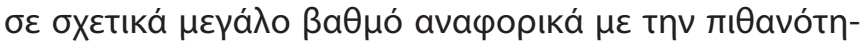

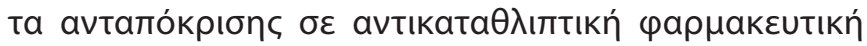

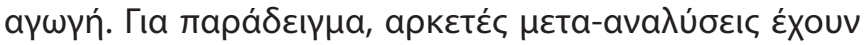

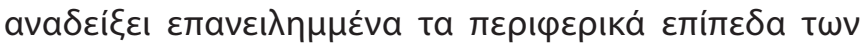
TNF-a, IL-6, IL-1 $\beta$, BDNF, IL-8 кaI CRP бтоv орó aípatoc,

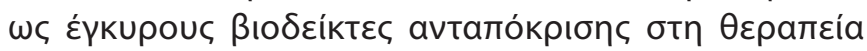

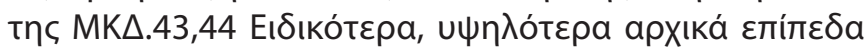

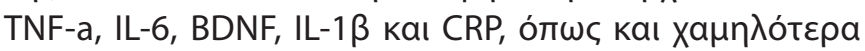

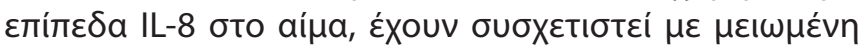

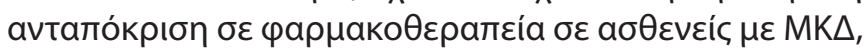

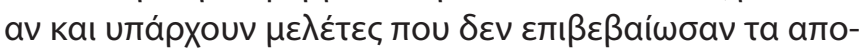

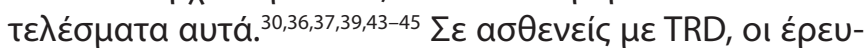

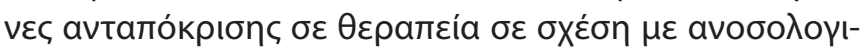

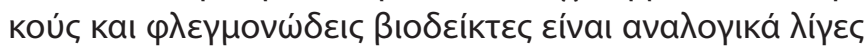

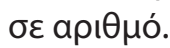

\section{a. $I L-1 \beta$}

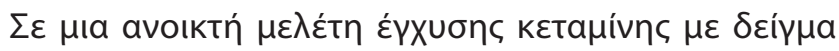

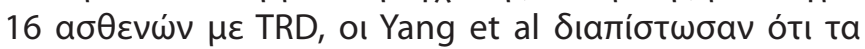

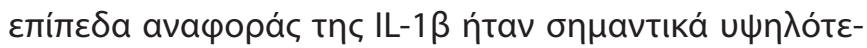

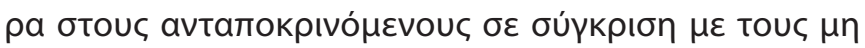

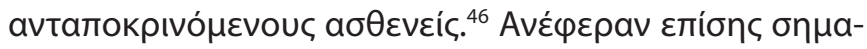

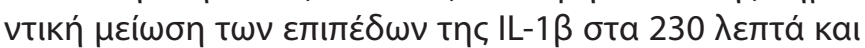

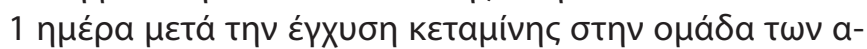

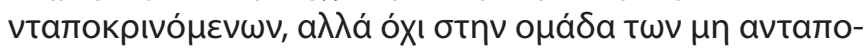

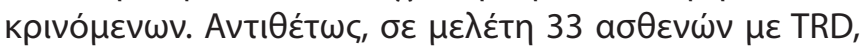

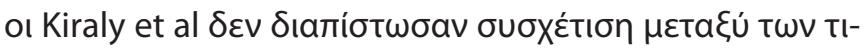

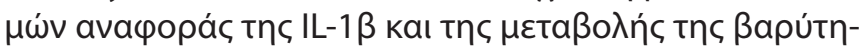

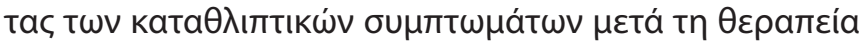

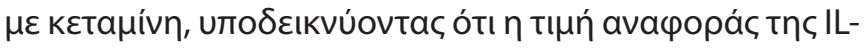

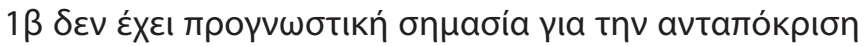

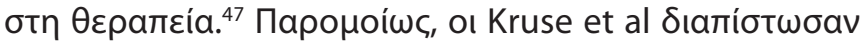

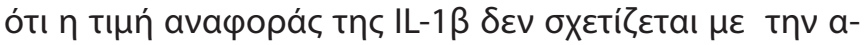

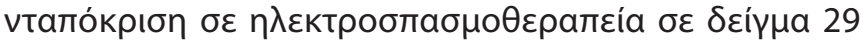

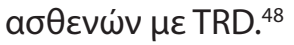




\section{$\beta$. IL-6}

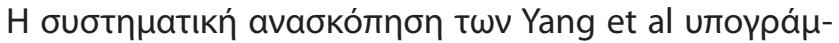

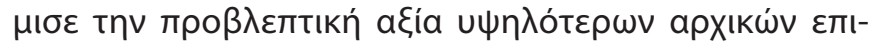

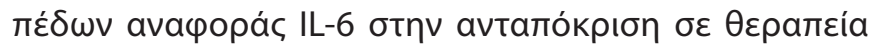

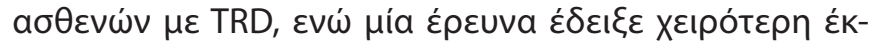

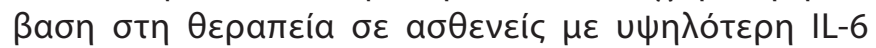

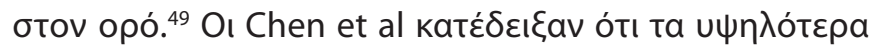

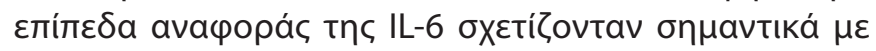

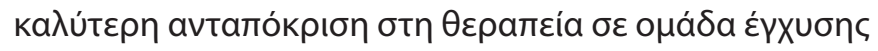

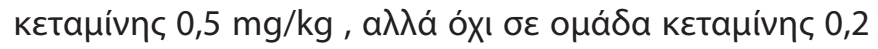

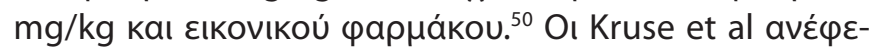

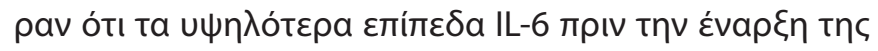

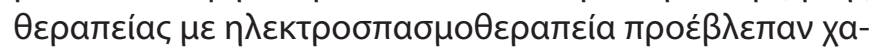

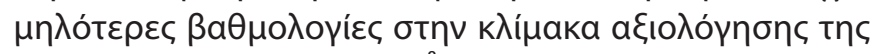

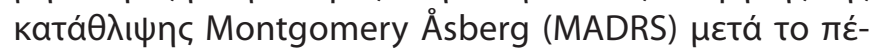

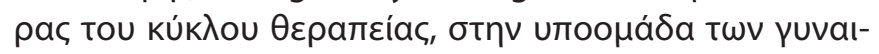

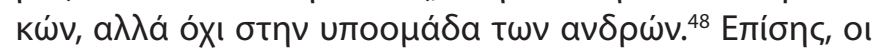

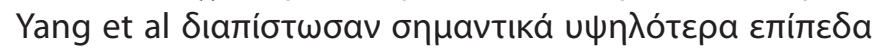

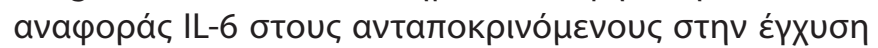

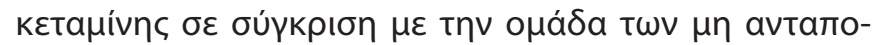

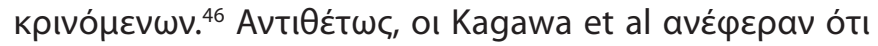

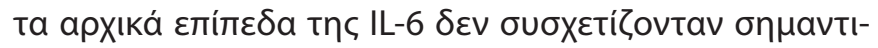

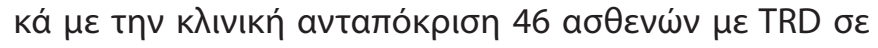

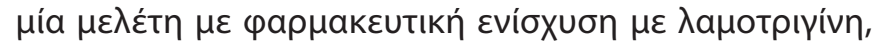

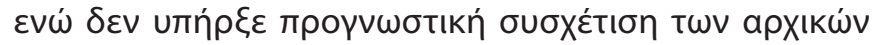

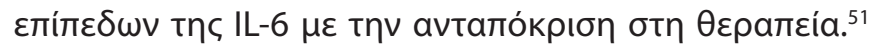

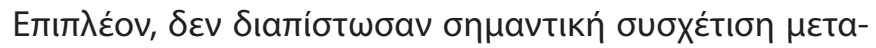

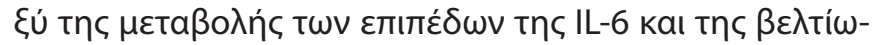

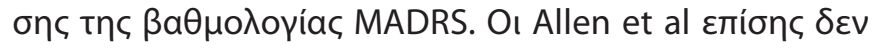

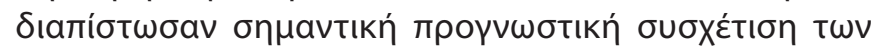

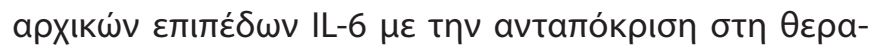

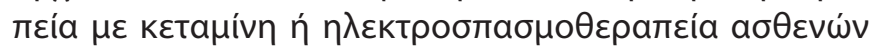

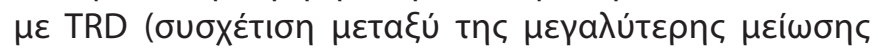

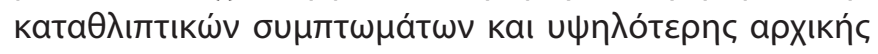

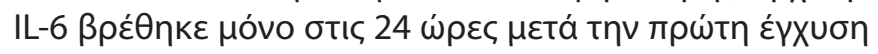

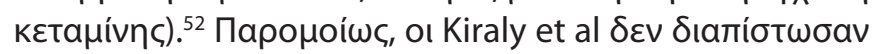

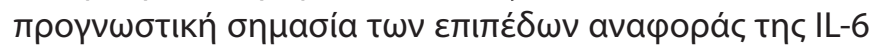

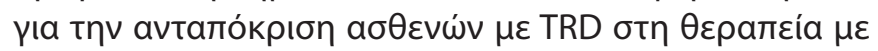

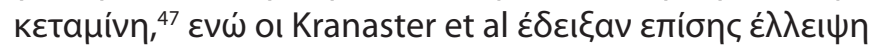

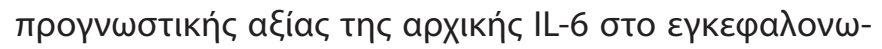

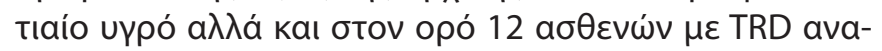

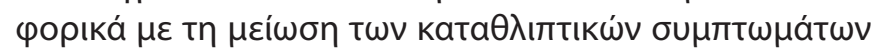

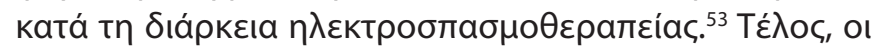

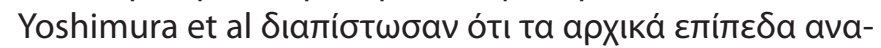

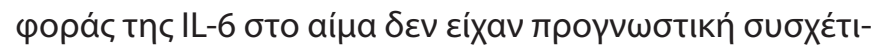

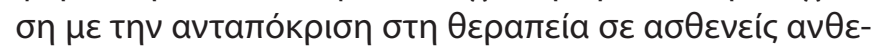

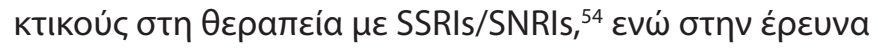

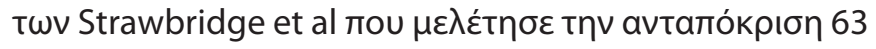

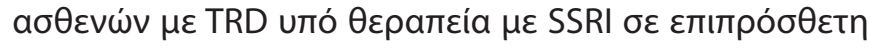

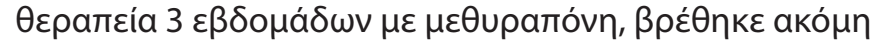

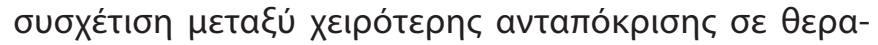

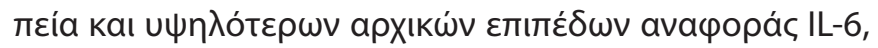

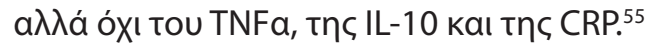

Y. IL-8

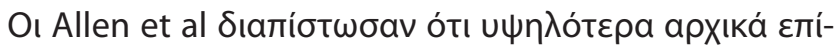

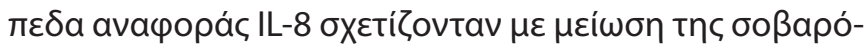

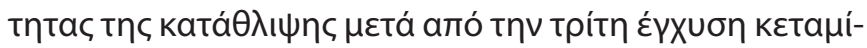

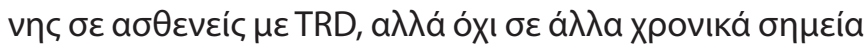

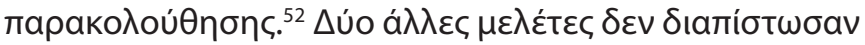

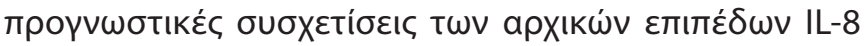

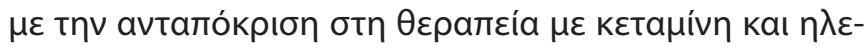

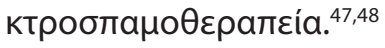

\section{ס. $I L-10$}

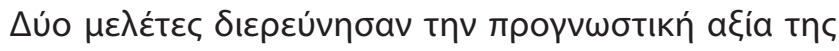

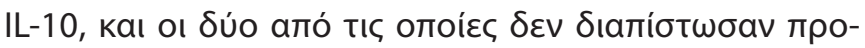

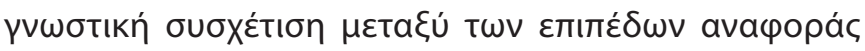

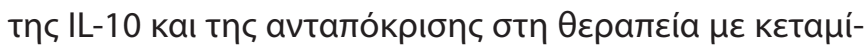
v $a \sigma \theta \varepsilon v \omega \dot{v} \mu \varepsilon \operatorname{TRD}^{47,52}$

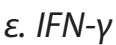

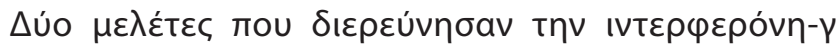

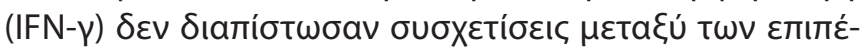

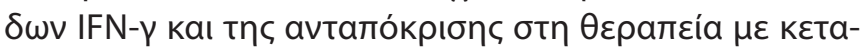

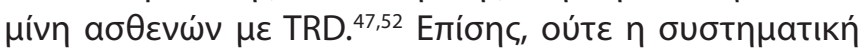

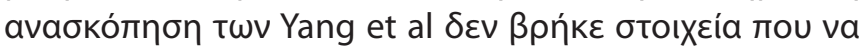

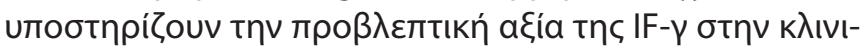

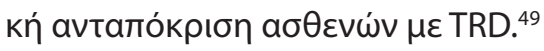

\section{$\sigma \tau . T N F-a$}

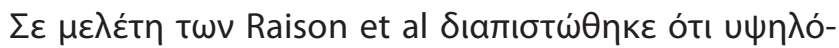

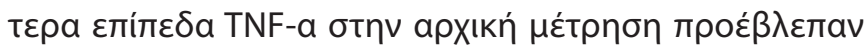

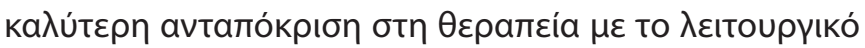

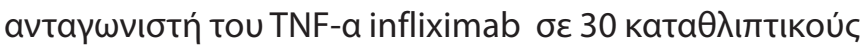

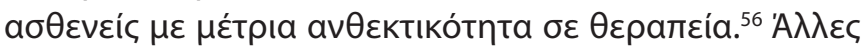

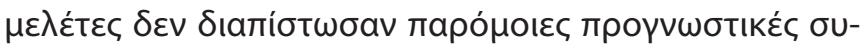

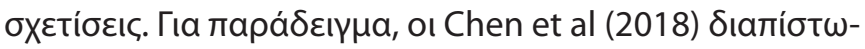

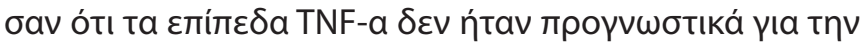

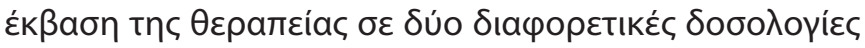

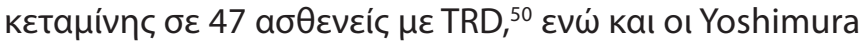

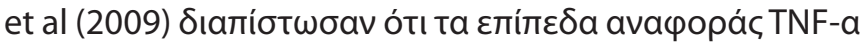

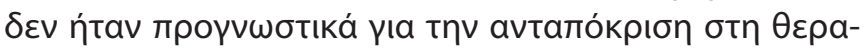

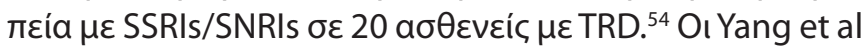

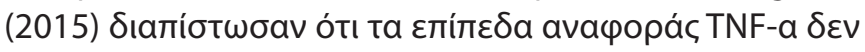

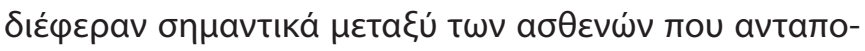

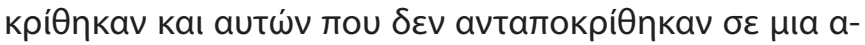

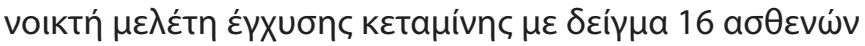
$\mu \varepsilon \operatorname{TRD}^{46} \varepsilon v \omega \dot{~ o l ~ K r u s e ~ e t ~ a l ~ k a ı ~ K i r a l y ~ e t ~ a l ~ \delta \varepsilon v ~ \delta ı a r i ́ o t \omega-~}$

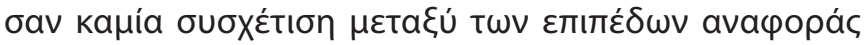

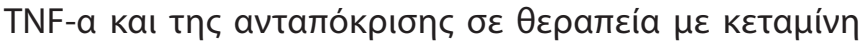

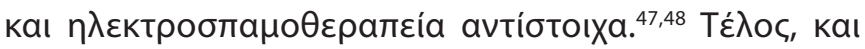

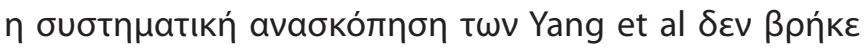

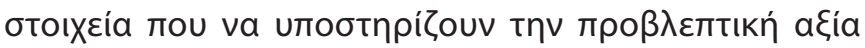

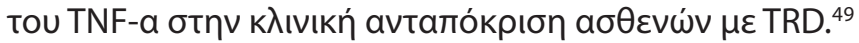




\section{ל. CRP}

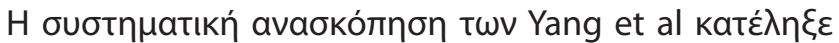

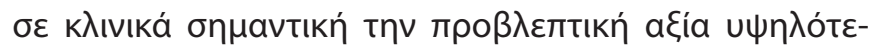

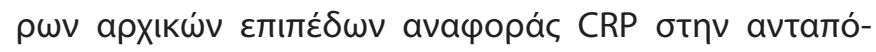

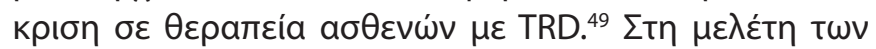

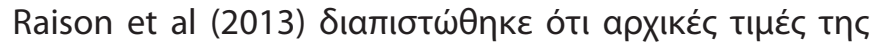

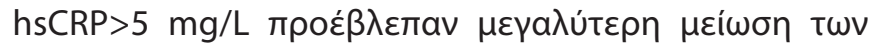

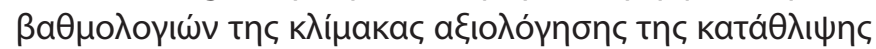

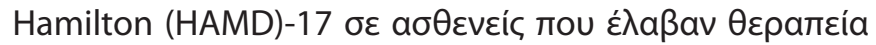

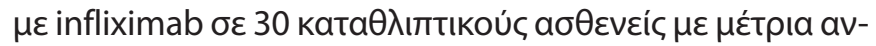

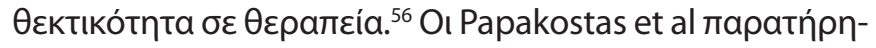

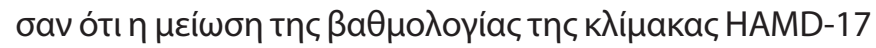

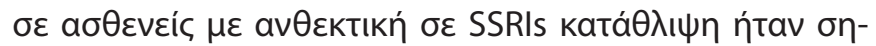

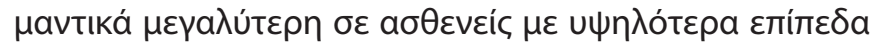

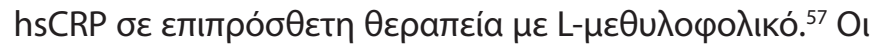

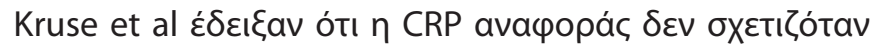

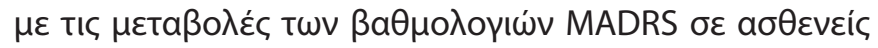

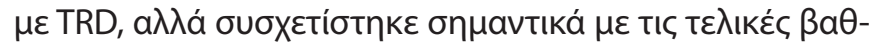

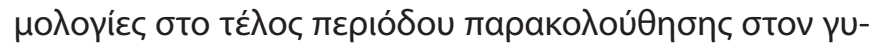

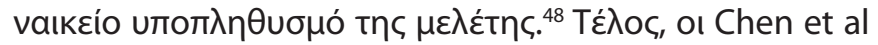

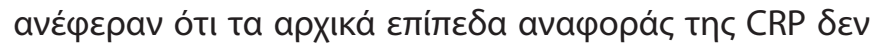

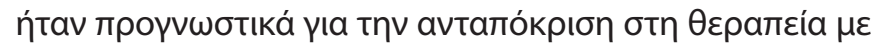

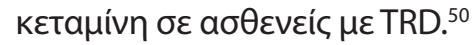

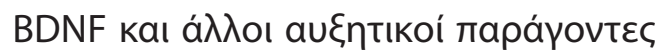

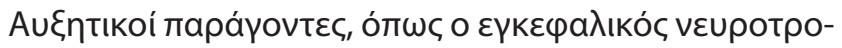

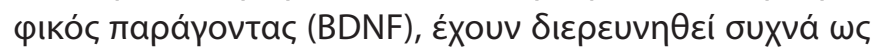

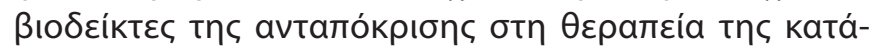

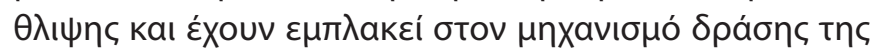

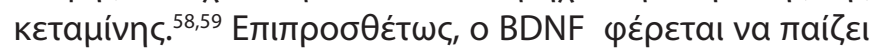

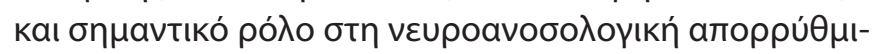

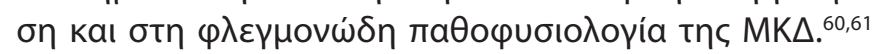

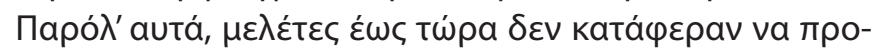

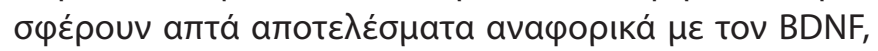

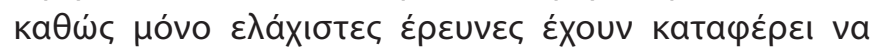

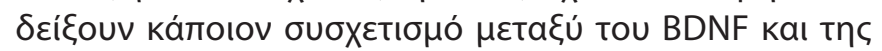

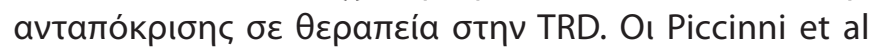

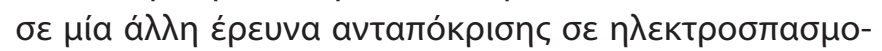

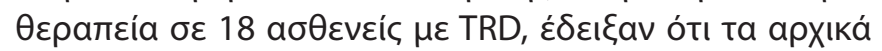

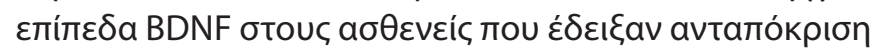

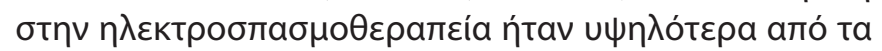

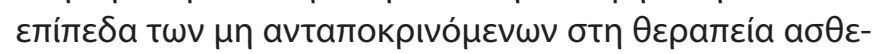

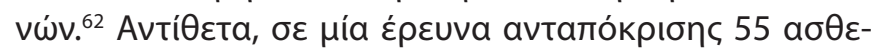

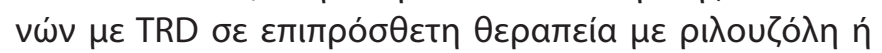

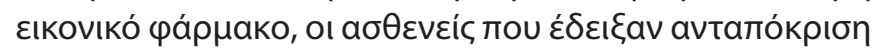

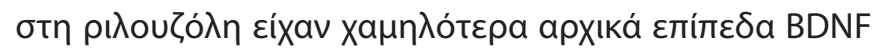

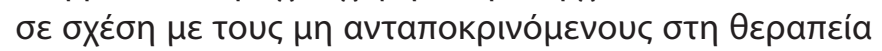

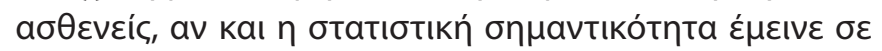

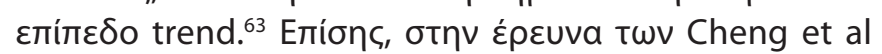

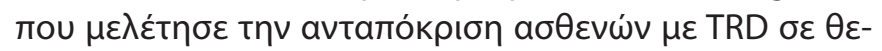

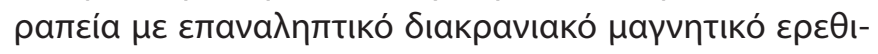

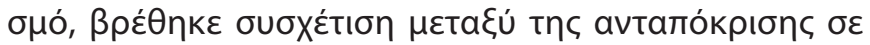

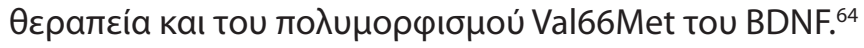

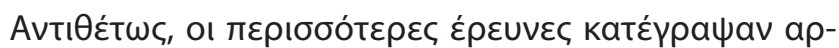

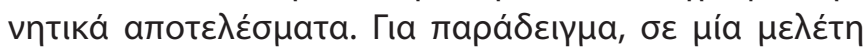

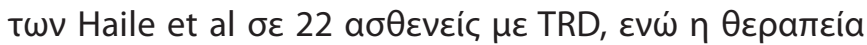

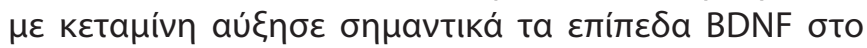

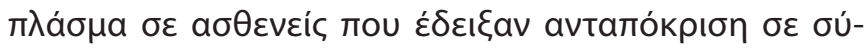

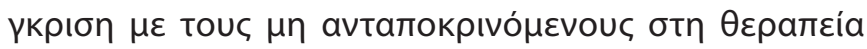

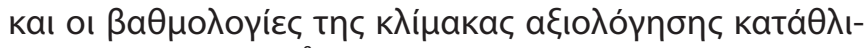

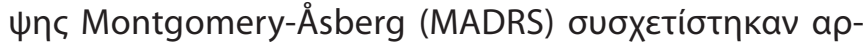

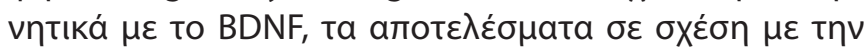

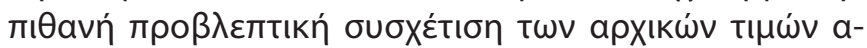

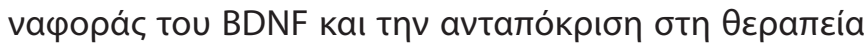

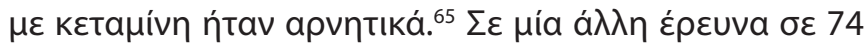

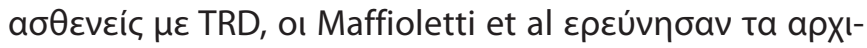

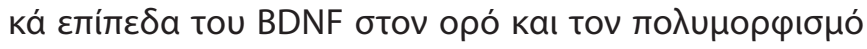

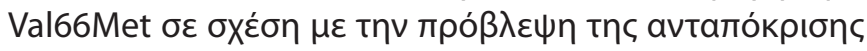

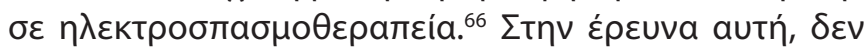

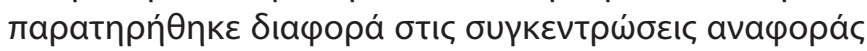

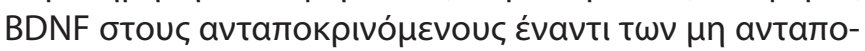

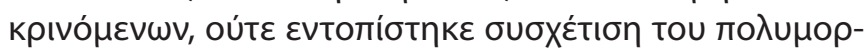

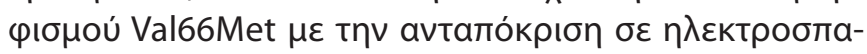

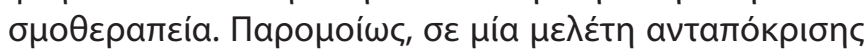

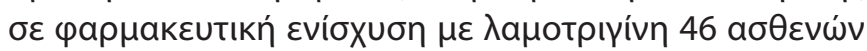

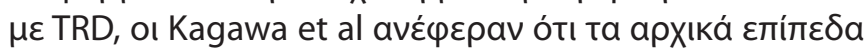

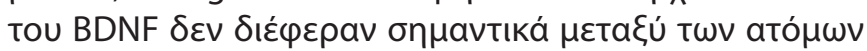

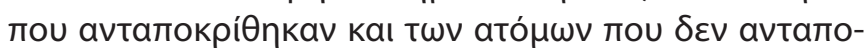

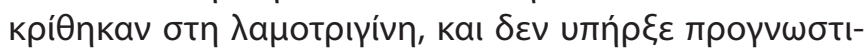

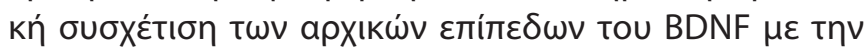

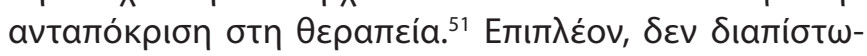

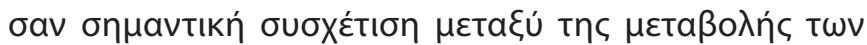

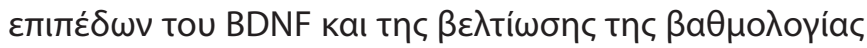

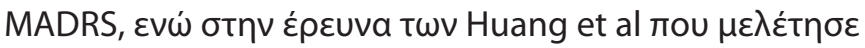

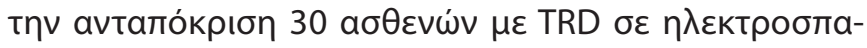

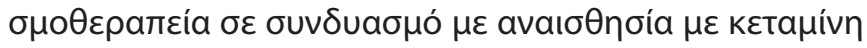

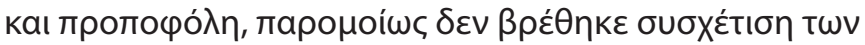

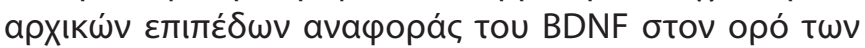

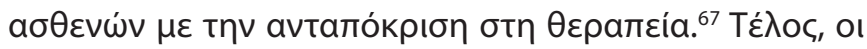

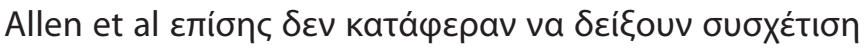

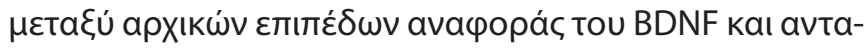

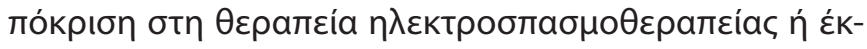

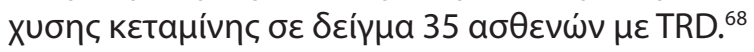

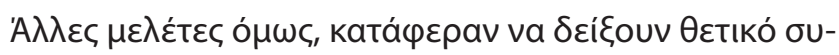

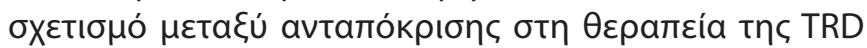

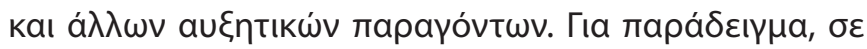

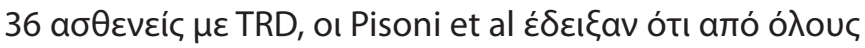

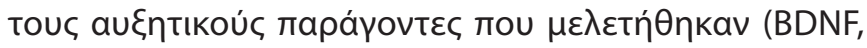

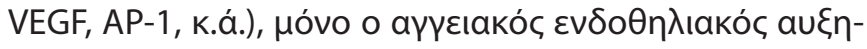

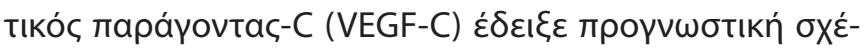

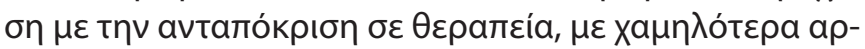

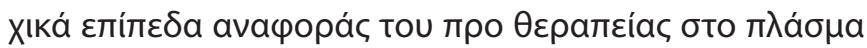

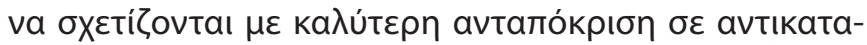

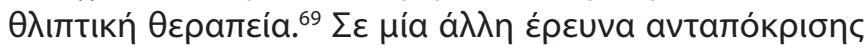




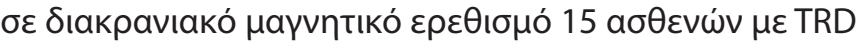

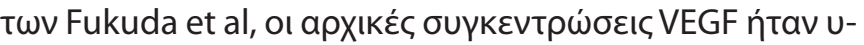

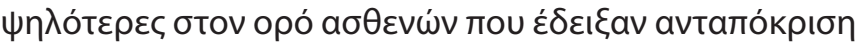

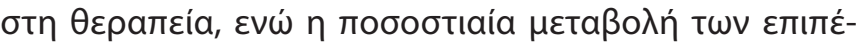

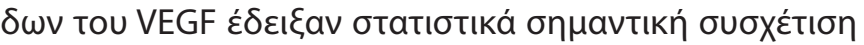

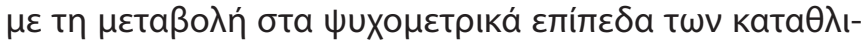

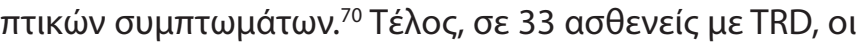

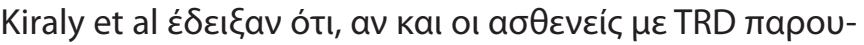

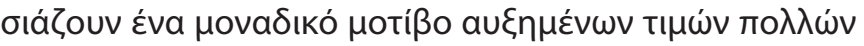

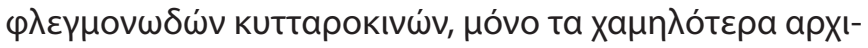

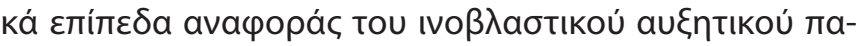

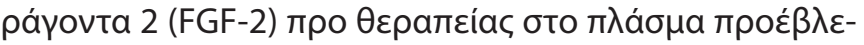

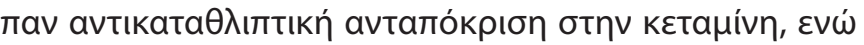

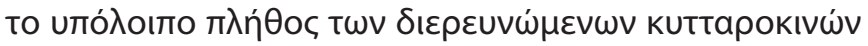

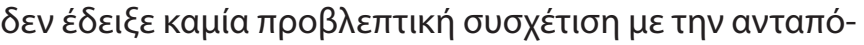

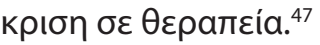

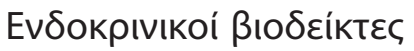

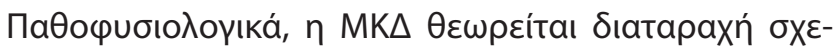

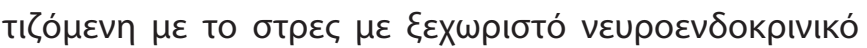

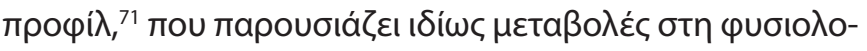

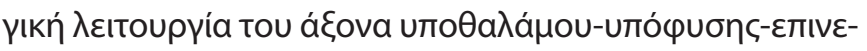

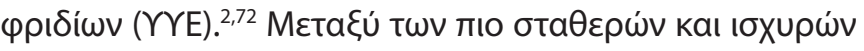

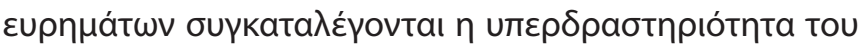

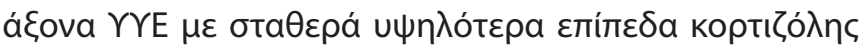

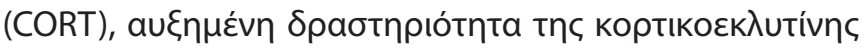

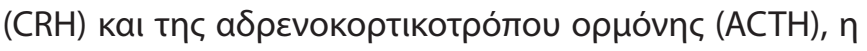

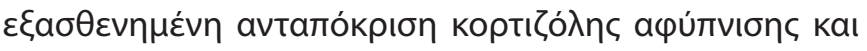

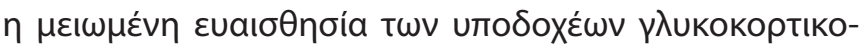

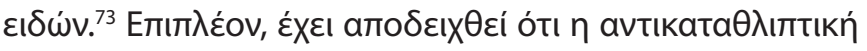

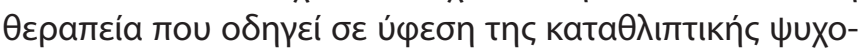

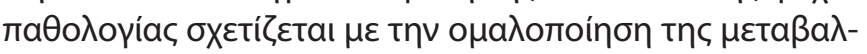

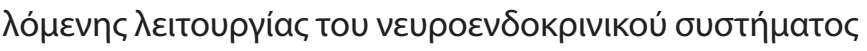

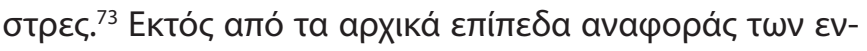

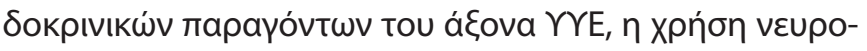

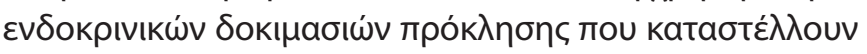

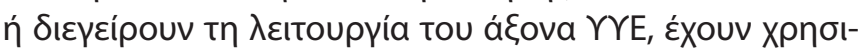

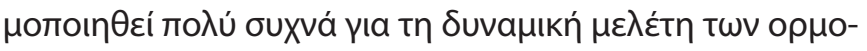

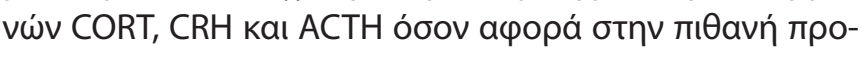

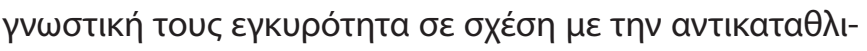

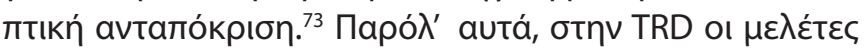

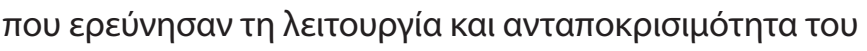

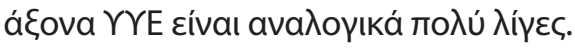

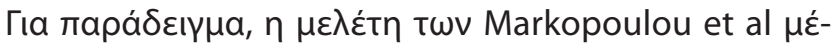

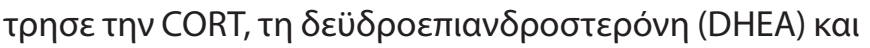

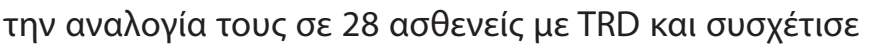

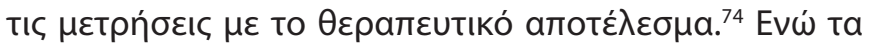

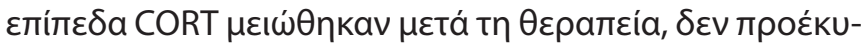

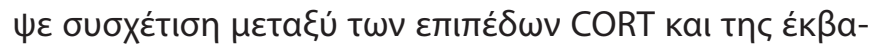

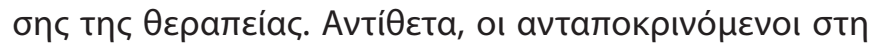

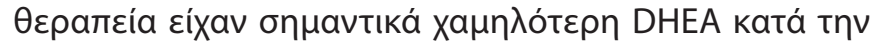

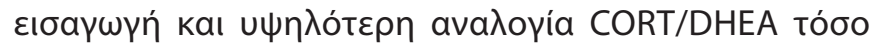

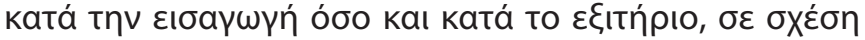

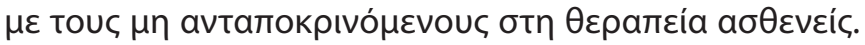

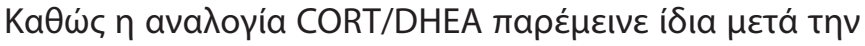

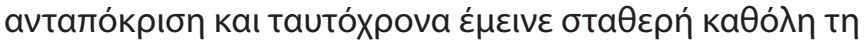

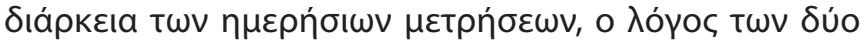

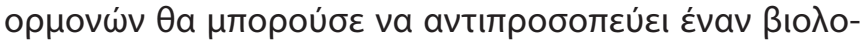

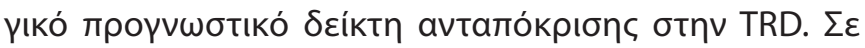

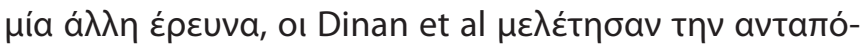

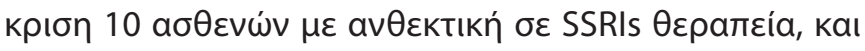

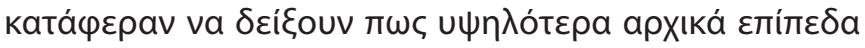

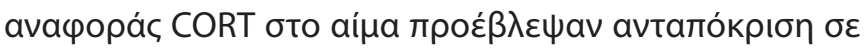

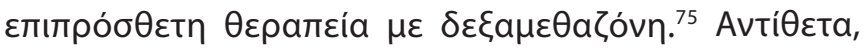

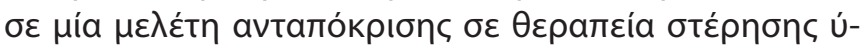

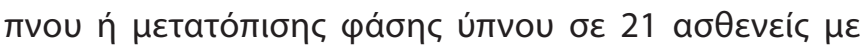

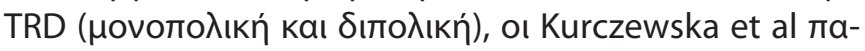

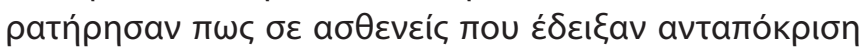

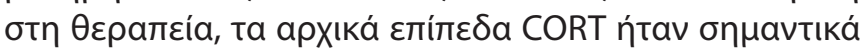

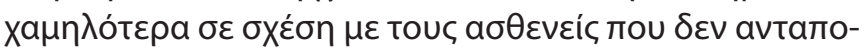
крї̈пкаv. ${ }^{76}$

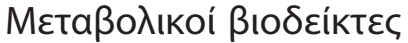

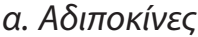

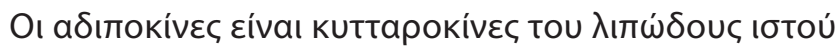

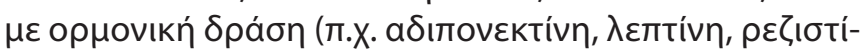

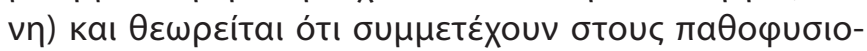

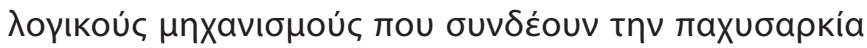

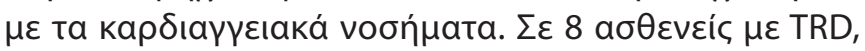

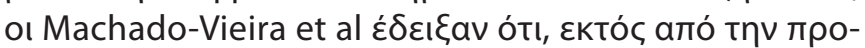

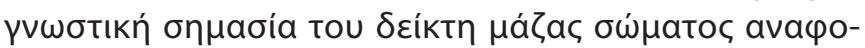

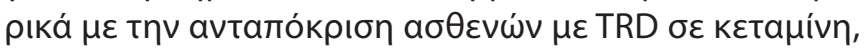

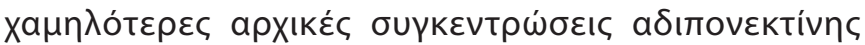

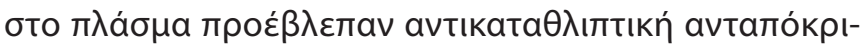

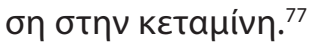

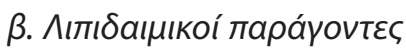

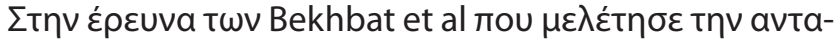

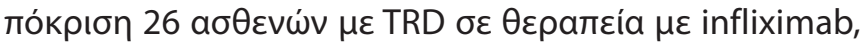

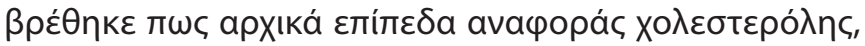

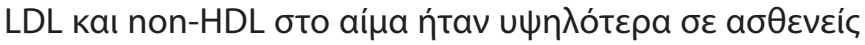

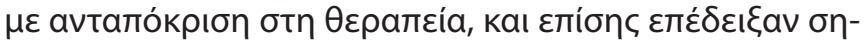

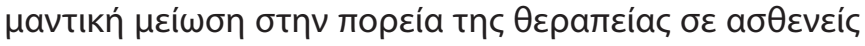

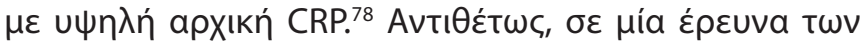

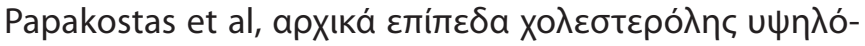

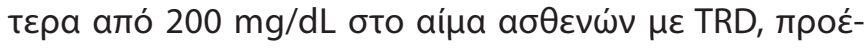

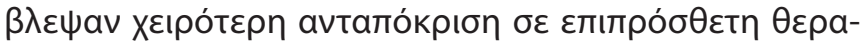

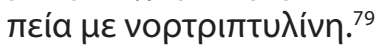

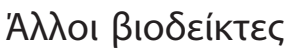

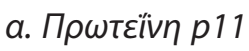

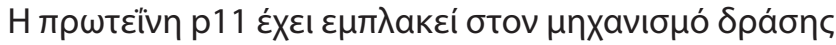

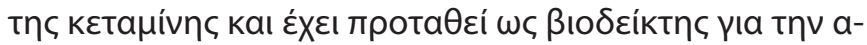

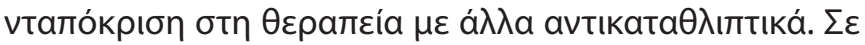




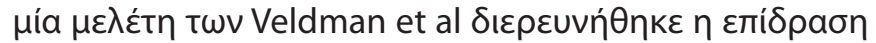

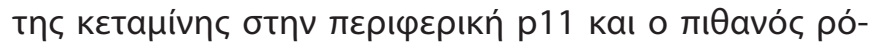

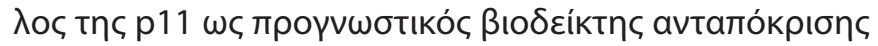

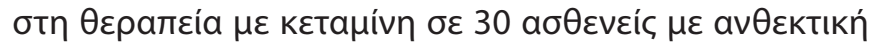

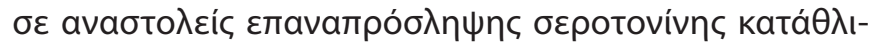

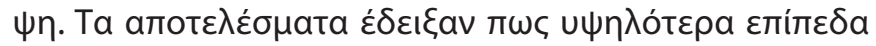

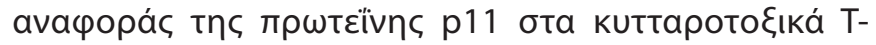

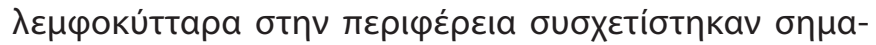

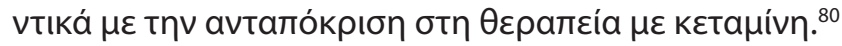

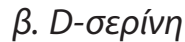

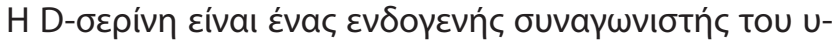

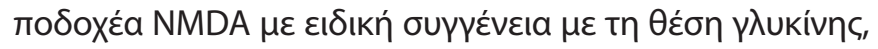

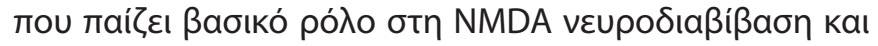

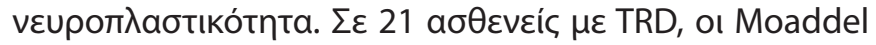

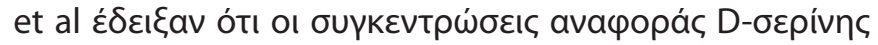

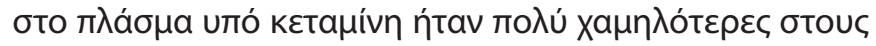

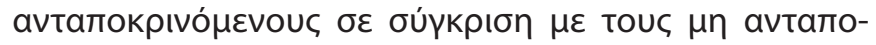

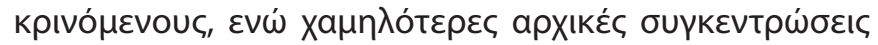

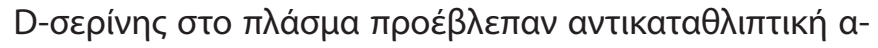

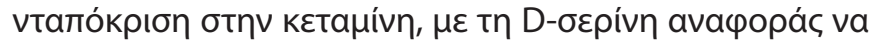

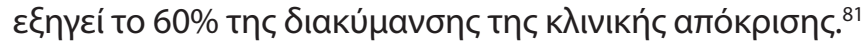

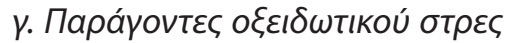

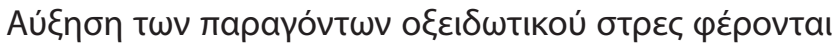

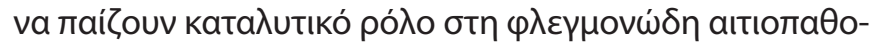

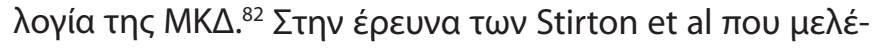

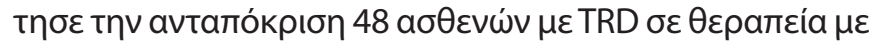

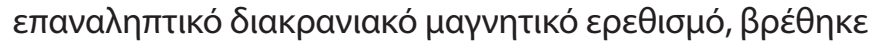

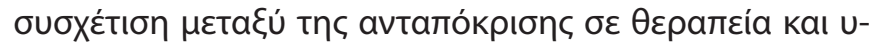

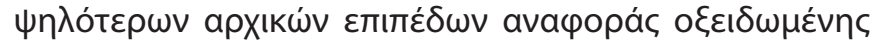

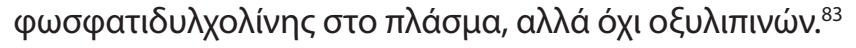

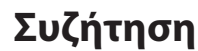

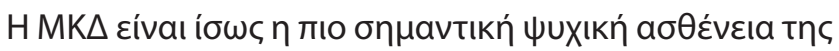

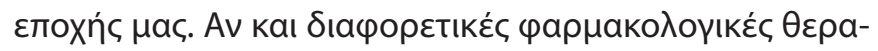

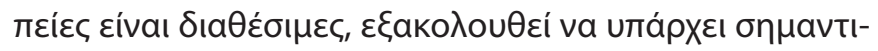

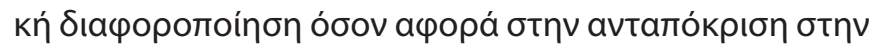

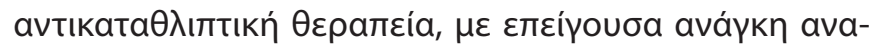

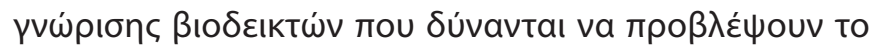

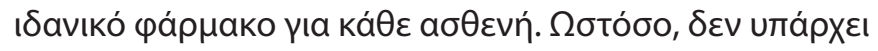

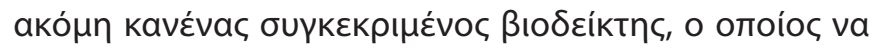

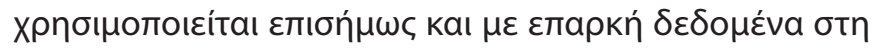

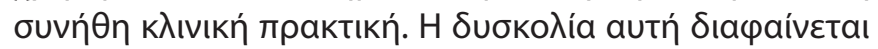

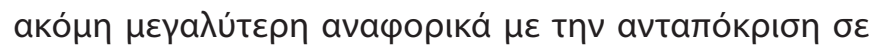

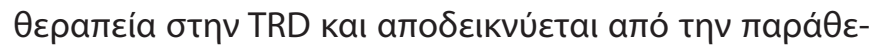

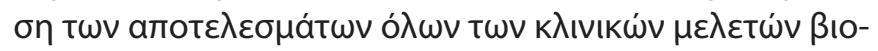

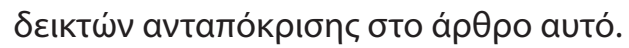

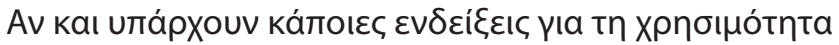

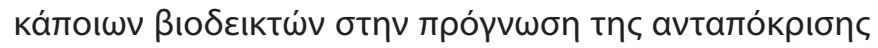

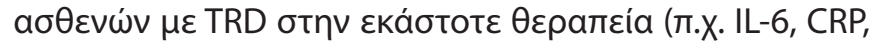

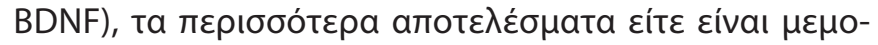

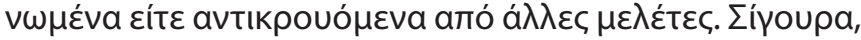

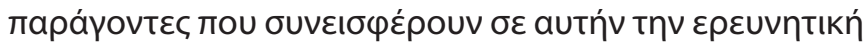

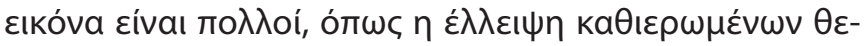

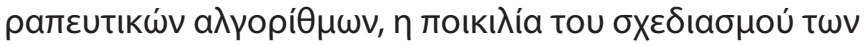

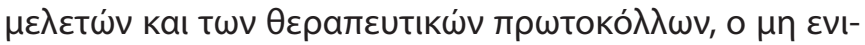

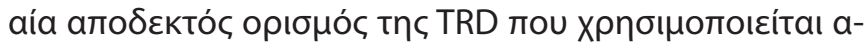

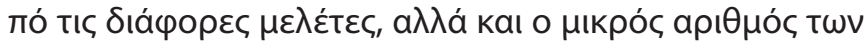

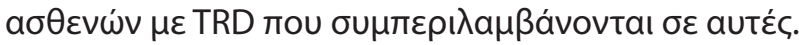

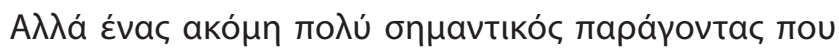

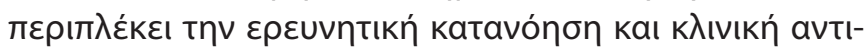

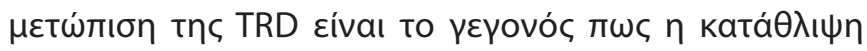

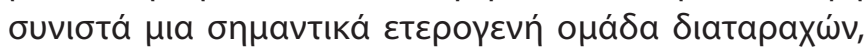

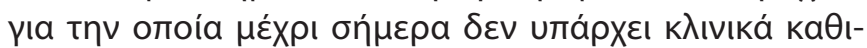

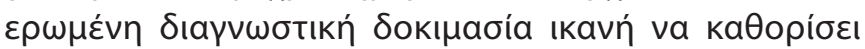

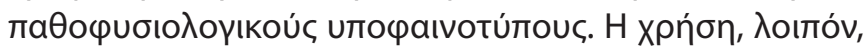

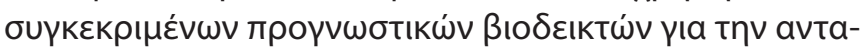

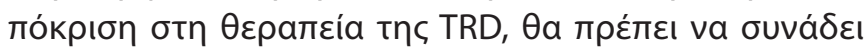

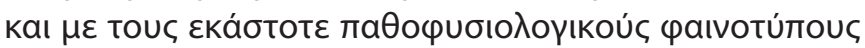

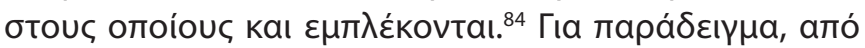

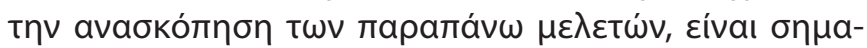

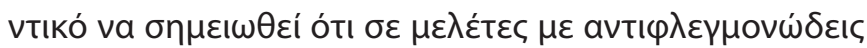

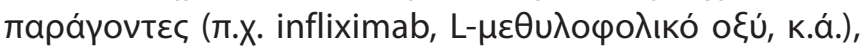

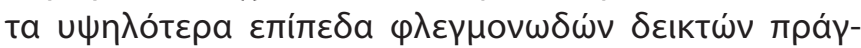

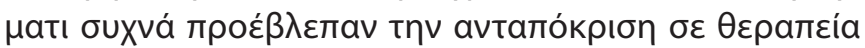

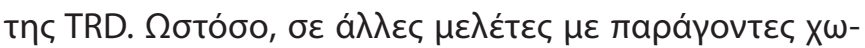

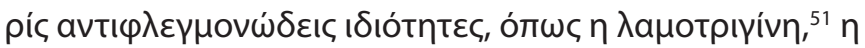

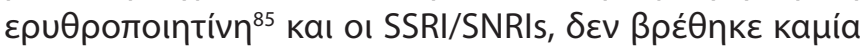

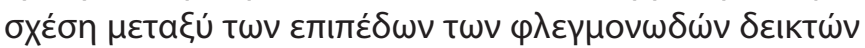

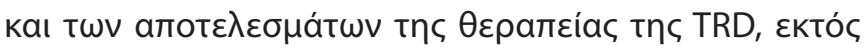

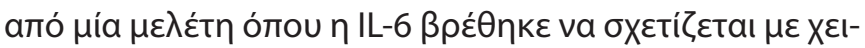

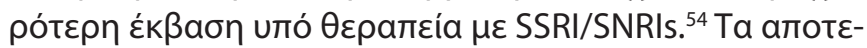

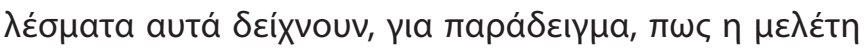

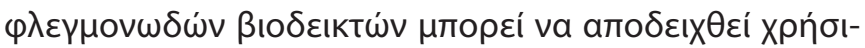

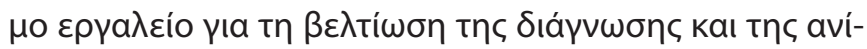

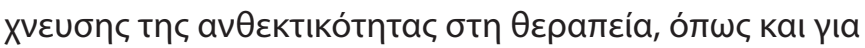

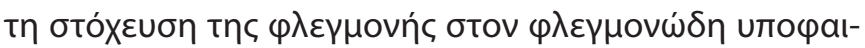

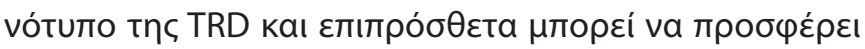

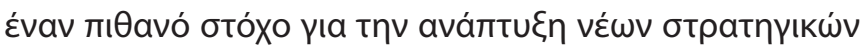

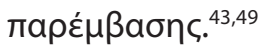

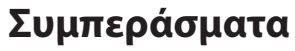

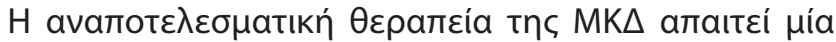

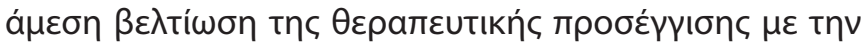

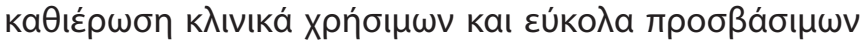

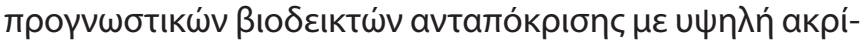

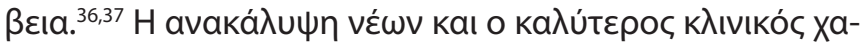

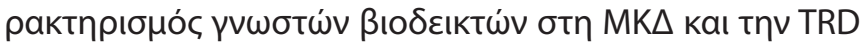

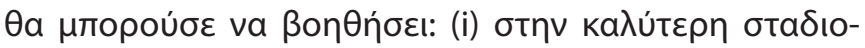

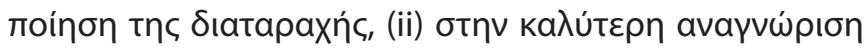

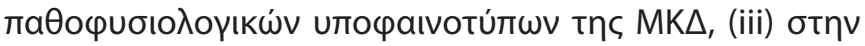

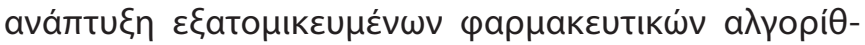

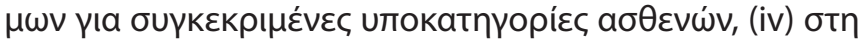




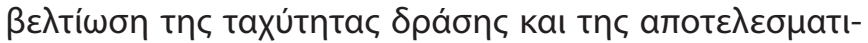

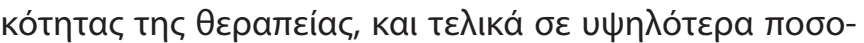

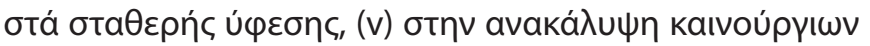

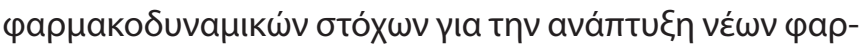

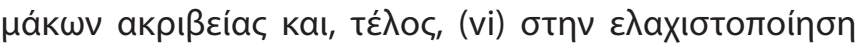

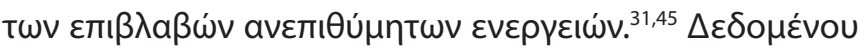

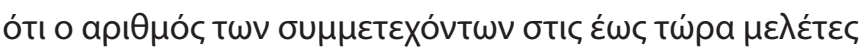

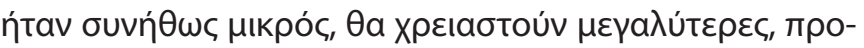

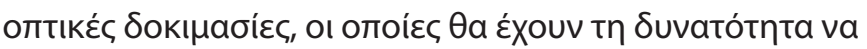

\section{BıBลtoypacpía}

1. Moussavi S, Chatterji S, Verdes E, Tandon A, Patel V, Ustun B. Depression, chronic diseases, and decrements in health: results from the World Health Surveys. Lancet 2007, 370:851-858, doi: S0140-6736(07)61415-9

2. Otte C, Gold SM, Penninx BW, Pariante CM, Etkin A, Fava M et al. Major depressive disorder. Nat Rev Dis Primers 2016, 2:16065, doi: 10.1038/ nrdp. 2016.65

3. Friedrich MJ. Depression Is the Leading Cause of Disability Around the World. JAMA 2017, 317:1517, doi: 10.1001/jama.2017.3826

4. American Psychiatric Association. Diagnostic and statistical manual of mental disorders. 5th ed. American Psychiatric Publishing, Arlington, VA, 2013, doi: 10.1176/appi.books.9780890425596

5. Turecki G, Brent DA, Gunnell D, O'Connor RC, Oquendo MA, Pirkis J et al. Suicide and suicide risk. Nat Rev Dis Primers 2019, 5:74, doi: 10.1038/ s41572-019-0121-0

6. Bachmann S. Epidemiology of Suicide and the Psychiatric Perspective. Int JEnviron Res Public Health 2018, 15:1425, doi: 10.3390/ijerph15071425

7. Fekadu A, Rane LJ, Wooderson SC, Markopoulou K, Poon L, Cleare AJ. Prediction of longer-term outcome of treatment-resistant depression in tertiary care. Br J Psychiatry 2012, 201:369-375, doi: 10.1192/ bjp.bp. 111.102665

8. Fekadu A, Wooderson SC, Markopoulo K, Donaldson C, Papadopoulos A, Cleare AJ. What happens to patients with treatment-resistant depression? A systematic review of medium to long term outcome studies. J Affect Disord 2009, 116:4-11, doi: 10.1016/j.jad.2008.10.014

9. Cuijpers P, Sijbrandij M, Koole SL, Andersson G, Beekman AT, Reynolds CF 3 rd. The efficacy of psychotherapy and pharmacotherapy in treating depressive and anxiety disorders: a meta-analysis of direct comparisons. World Psychiatry 2013, 12:137-148, doi: 10.1002/wps.20038

10. Cipriani A, Furukawa TA, Salanti G, Chaimani A, Atkinson LZ, Ogawa $Y$ et al. Comparative efficacy and acceptability of 21 antidepressant drugs for the acute treatment of adults with major depressive disorder: a systematic review and network meta-analysis. Lancet 2018, 391:1357-1366, doi: 10.1016/S0140-6736(17)32802-7

11. Cleare A, Pariante CM, Young AH, Anderson IM, Christmas D, Cowen PJ et al. Evidence-based guidelines for treating depressive disorders with antidepressants: A revision of the 2008 British Association for Psychopharmacology guidelines. J Psychopharmacol 2015, 29:459-525, doi: $10.1177 / 0269881115581093$

12. Rush AJ, Trivedi MH, Wisniewski SR, Nierenberg AA, Stewart JW, Warden $D$ et al. Acute and longer-term outcomes in depressed outpatients requiring one or several treatment steps: a $S T A R^{*} D$ report. Am J Psychiatry 2006, 163:1905-1917, doi: 10.1176/ajp.2006.163.11.1905

13. Fava M, Davidson KG. Definition and epidemiology of treatment-resistant depression. Psychiatr Clin North Am 1996, 19:179-200, doi: 10.1016/s0193-953x(05)70283-5

14. Zhdanava M, Pilon D, Ghelerter I, Chow W, Joshi K, Lefebvre P et al. The Prevalence and National Burden of Treatment-Resistant Depression

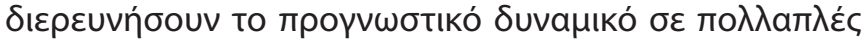

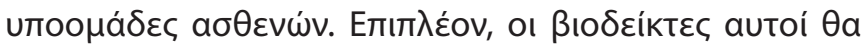

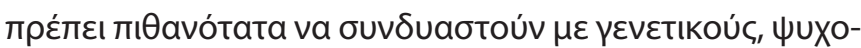

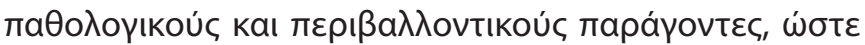

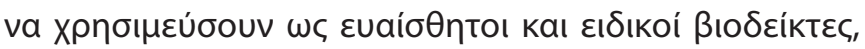

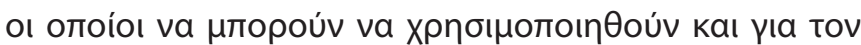

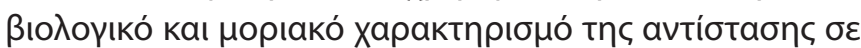

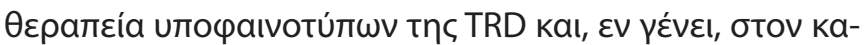

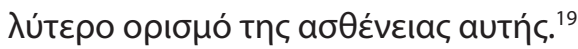

and Major Depressive Disorder in the United States. $J$ Clin Psychiatry 2021, 82:20m13699, doi: 10.4088/JCP.20m13699

15. Gillain B, Degraeve G, Dreesen T, De Bruecker G, Buntinx E, Beke D et al. Real-World Treatment Patterns, Outcomes, Resource Utilization and Costs in Treatment-Resistant Major Depressive Disorder: PATTERN, a Retrospective Cohort Study in Belgium. Pharmacoecon Open 2021, doi: 10.1007/s41669-021-00306-2

16. Demyttenaere K, Van Duppen Z. The Impact of (the Concept of) Treatment-Resistant Depression: An Opinion Review. Int J Neuropsychopharmacol 2019, 22:85-92, doi: 10.1093/ijnp/pyy052

17. Heerlein K, De Giorgi S, Degraeve G, Frodl T, Hagedoorn W, OliveiraMaia AJ et al. Real-World Evidence from a European Cohort Study of Patients with Treatment Resistant Depression: Healthcare Resource Utilization: Healthcare resource utilization in TRD. J Affect Disord 2021, doi:10.1016/j.jad.2021.11.004

18. Heimann $\mathrm{H}$. Therapy-resistant depressions: symptoms and syndromes. Contributions to symptomatology and syndromes. Pharmakopsychiatr Neuropsychopharmakol 1974, 7:139-144, doi: 10.1055/s-0028-1094412

19. Sforzini L. Lost in translation. The quest for definitions of treatment-resistant depression with a focus on inflammation-related gene expression. Brain Behav Immun Health 2021, 16:100331, doi: 10.1016/j. bbih.2021.100331

20. Trevino K, McClintock SM, McDonald Fischer N, Vora A, Husain MM. Defining treatment-resistant depression: a comprehensive review of the literature. Ann Clin Psychiatry 2014, 26:222-232, PMID: 25166485

21. Gaynes BN, Lux L, Gartlehner G, Asher G, Forman-Hoffman V, Green $J$ et al. Defining treatment-resistant depression. Depress Anxiety 2020, 37:134-145, doi: 10.1002/da.22968

22. Brown S, Rittenbach K, Cheung S, McKean G, MacMaster FP, Clement F. Current and Common Definitions of Treatment-Resistant Depression: Findings from a Systematic Review and Qualitative Interviews. Can J Psychiatry 2019, 64:380-387, doi: 10.1177/0706743719828965

23. Han C, Wang G, Chan S, Kato T, Ng CH, Tan W et al. Definition and Identification of Patients with Treatment-Resistant Depression in Real-World Clinical Practice Settings Across Asia. Neuropsychiatr Dis Treat 2020, 16:2929-2941, doi: 10.2147/NDT.S264799

24. Malhi GS, Das P, Mannie Z, Irwin L. Treatment-resistant depression: problematic illness or a problem in our approach? Br J Psychiatry 2019, 214:1-3, doi: 10.1192/bjp.2018.246

25. Ruberto VL, Jha MK, Murrough JW. Pharmacological Treatments for Patients with Treatment-Resistant Depression. Pharmaceuticals (Basel) 2020, 13:116, doi: 10.3390/ph13060116

26. Pandarakalam JP. Challenges of Treatment-resistant Depression. Psychiatr Danub 2018, 30:273-284, doi: 10.24869/psyd.2018.273

27. Rush AJ, Kraemer HC, Sackeim HA, Fava M, Trivedi MH, Frank E et al. Report by the ACNP Task Force on response and remission in major depressive disorder. Neuropsychopharmacology 2006, 31:1841-1853, doi: 10.1038/sj.npp.1301131 
28. Kennedy SH, Lam RW, McIntyre RS, Tourjman SV, Bhat V, Blier P et al. Canadian Network for Mood and Anxiety Treatments (CANMAT) 2016 Clinical Guidelines for the Management of Adults with Major Depressive Disorder: Section 3. Pharmacological Treatments. Can J Psychiatry 2016, 61:540-560, doi: 10.1177/0706743716659417

29. Nierenberg AA. Predictors of response to antidepressants general principles and clinical implications. Psychiatr Clin North Am 2003, 26 : 345-352, viii, doi: 10.1016/s0193-953x(02)00105-3

30. Chan MK, Cooper JD, Bot M, Birkenhager TK, Bergink V, Drexhage $\mathrm{HA}$ et al. Blood-based immune-endocrine biomarkers of treatment response in depression. J Psychiatr Res 2016, 83:249-259, doi: 10.1016/j. jpsychires.2016.08.020

31. Chan MK, Gottschalk MG, Haenisch F, Tomasik J, Ruland T, Rahmoune $\mathrm{H}$ et al. Applications of blood-based protein biomarker strategies in the study of psychiatric disorders. Prog Neurobiol 2014, 122:45-72, doi: 10.1016/j.pneurobio.2014.08.002

32. Bennabi D, Aouizerate B, El-Hage W, Doumy O, Moliere F, Courtet $P$ et al. Risk factors for treatment resistance in unipolar depression: a systematic review. J Affect Disord 2015, 171:137-141, doi: 10.1016/j. jad.2014.09.020

33. Miller DB, O'Callaghan JP. Personalized medicine in major depressive disorder - opportunities and pitfalls. Metabolism 2013, 62(Suppl 1): S34-39, doi: 10.1016/j.metabol.2012.08.021

34. Biomarkers Definitions Working Group. Biomarkers and surrogate endpoints: preferred definitions and conceptual framework. Clin Pharmacol Ther 2001, 69:89-95, doi: 10.1067/mcp.2001.113989

35. Lozupone M, La Montagna M, D'Urso F, Daniele A, Greco A, Seripa D et al. The Role of Biomarkers in Psychiatry. Adv Exp Med Biol 2019, 1118:135-162, doi: 10.1007/978-3-030-05542-4_7

36. Gadad BS, Jha MK, Czysz A, Furman JL, Mayes TL, Emslie MP et al. Peripheral biomarkers of major depression and antidepressant treatment response: Current knowledge and future outlooks. J Affect Disord 2018, 233:3-14, doi: 10.1016/j.jad.2017.07.001

37. Mora C, Zonca V, Riva MA, Cattaneo A. Blood biomarkers and treatment response in major depression. Expert Rev Mol Diagn 2018, 18:513-529, doi: 10.1080/14737159.2018.1470927

38. Jani BD, McLean G, Nicholl BI, Barry SJ, Sattar N, Mair FS et al. Risk assessment and predicting outcomes in patients with depressive symptoms: a review of potential role of peripheral blood based biomarkers. Front Hum Neurosci 2015, 9:18, doi: 10.3389/fnhum.2015.00018

39. Busch Y, Menke A. Blood-based biomarkers predicting response to antidepressants. J Neural Transm (Vienna) 2019, 126:47-63, doi: 10.1007/s00702-018-1844-x

40. Miller AH, Raison CL. The role of inflammation in depression: from evolutionary imperative to modern treatment target. Nat Rev Immunol 2016, 16:22-34, doi: 10.1038/nri.2015.5

41. Köhler-Forsberg O, N Lydholm C, Hjorthøj C, Nordentoft M, Mors O, Benros ME. Efficacy of anti-inflammatory treatment on major depressive disorder or depressive symptoms: meta-analysis of clinical trials. Acta Psychiatr Scand 2019, 139:404-419, doi: 10.1111/acps.13016

42. Beurel E, Toups M, Nemeroff CB. The Bidirectional Relationship of Depression and Inflammation: Double Trouble. Neuron 2020, 107:234256, doi: 10.1016/j.neuron.2020.06.002

43. Strawbridge R, Arnone D, Danese A, Papadopoulos A, Herane Vives A, Cleare AJ. Inflammation and clinical response to treatment in depression: A meta-analysis. Eur Neuropsychopharmacol 2015, 25:1532-1543, doi: 10.1016/j.euroneuro.2015.06.007

44. Liu JJ, Wei YB, Strawbridge R, Bao Y, Chang S, Shi L et al. Peripheral cytokine levels and response to antidepressant treatment in depression: a systematic review and meta-analysis. Mol Psychiatry 2020, 25:339-350, doi: 10.1038/s41380-019-0474-5
45. Strawbridge R, Young AH, Cleare AJ. Biomarkers for depression: recent insights, current challenges and future prospects. Neuropsychiatr Dis Treat 2017, 13:1245-1262, doi: 10.2147/NDT.S114542

46. Yang JJ, Wang N, Yang C, Shi JY, Yu HY, Hashimoto K. Serum interleukin-6 is a predictive biomarker for ketamine's antidepressant effect in treatment-resistant patients with major depression. Biol Psychiatry 2015, 77:e19-e20, doi: 10.1016/j.biopsych.2014.06.021

47. Kiraly DD, Horn SR, Van Dam NT, Costi S, Schwartz J, Kim-Schulze S et al. Altered peripheral immune profiles in treatment-resistant depression: response to ketamine and prediction of treatment outcome. Transl Psychiatry 2017, 7:e1065, doi: 10.1038/tp.2017.31

48. Kruse JL, Congdon E, Olmstead R, Njau S, Breen EC, Narr KL et al. Inflammation and Improvement of Depression Following Electroconvulsive Therapy in Treatment-Resistant Depression. J Clin Psychiatry 2018, 79:17m11597, doi: 10.4088/JCP.17m11597

49. Yang C, Wardenaar KJ, Bosker FJ, Li J, Schoevers RA. Inflammatory markers and treatment outcome in treatment resistant depression: A systematic review. J Affect Disord 2019, 257:640-649, doi:10.1016/j. jad.2019.07.045

50. Chen MH, Li CT, Lin WC, Hong CJ, Tu PC, Bai YM et al. Rapid inflammation modulation and antidepressant efficacy of a low-dose ketamine infusion in treatment-resistant depression: A randomized, double-blind control study. Psychiatry Res 2018, 269:207-211, doi: 10.1016/j.psychres.2018.08.078

51. Kagawa S, Mihara K, Suzuki T, Nagai G, Nakamura A, Nemoto K et al. Both Serum Brain-Derived Neurotrophic Factor and Interleukin-6 Levels Are Not Associated with Therapeutic Response to Lamotrigine Augmentation Therapy in Treatment-Resistant Depressive Disorder. Neuropsychobiology 2017, 75:145-150, doi: 10.1159/000484665

52. Allen AP, Naughton M, Dowling J, Walsh A, O'Shea R, Shorten G et al. Kynurenine pathway metabolism and the neurobiology of treatment-resistant depression: Comparison of multiple ketamine infusions and electroconvulsive therapy. J Psychiatr Res 2018, 100:24-32, doi: 10.1016/j.jpsychires.2018.02.011

53. Kranaster L, Hoyer C, Aksay SS, Bumb JM, Müller N, Zill P et al. Biomarkers for Antidepressant Efficacy of Electroconvulsive Therapy: An Exploratory Cerebrospinal Fluid Study. Neuropsychobiology 2019, 77:13-22, doi: 10.1159/000491401

54. Yoshimura R, Hori H, Ikenouchi-Sugita A, Umene-Nakano W, Ueda N, Nakamura J. Higher plasma interleukin-6 (IL-6) level is associated with SSRI- or SNRI-refractory depression. Prog Neuropsychopharmacol Biol Psychiatry 2009, 33:722-726, doi: 10.1016/j.pnpbp.2009.03.020

55. Strawbridge R, Jamieson A, Hodsoll J, Ferrier IN, McAllister-Williams $\mathrm{RH}$, Powell TR et al. The Role of Inflammatory Proteins in AntiGlucocorticoid Therapy for Treatment-Resistant Depression. J Clin Med 2021, 10:784, doi: 10.3390/jcm10040784

56. Raison CL, Rutherford RE, Woolwine BJ, Shuo C, Schettler P, Drake DF et al. A randomized controlled trial of the tumor necrosis factor antagonist infliximab for treatment-resistant depression: the role of baseline inflammatory biomarkers. JAMA Psychiatry 2013, 70:31-41, doi: 10.1001/2013.jamapsychiatry.4

57. Papakostas GI, Shelton RC, Zajecka JM, Bottiglieri T, Roffman J, Cassiello $C$ et al. Effect of adjunctive L-methylfolate $15 \mathrm{mg}$ among inadequate responders to SSRIs in depressed patients who were stratified by biomarker levels and genotype: results from a randomized clinical trial. J Clin Psychiatry 2014, 75:855-863, doi: 10.4088/ JCP.13m08947

58. Ochi T, Vyalova NM, Losenkov IS, Levchuk LA, Osmanova DZ, Mikhalitskaya EV et al. Investigating the potential role of BDNF and PRL genotypes on antidepressant response in depression patients: $A$ prospective inception cohort study in treatment-free patients. $J$ Affect Disord 2019, 259:432-439, doi: 10.1016/j.jad.2019.08.058 
59. Li S, Luo X, Hua D, Wang Y, Zhan G, Huang N et al. Ketamine Alleviates Postoperative Depression-Like Symptoms in Susceptible Mice: The Role of BDNF-TrkB Signaling. Front Pharmacol 2019, 10:1702, doi: 10.3389/fphar.2019.01702

60. Jin Y, Sun LH, Yang W, Cui RJ, Xu SB. The Role of BDNF in the Neuroimmune Axis Regulation of Mood Disorders. Front Neurol 2019, 10:515, doi: 10.3389/fneur.2019.00515

61. Zhang JC, Yao W, Hashimoto K. Brain-derived Neurotrophic Factor (BDNF)-TrkB Signaling in Inflammation-related Depression and Potential Therapeutic Targets. Curr Neuropharmacol 2016, 14:721-731, doi: 10.2174/1570159x14666160119094646

62. Piccinni A, Del Debbio A, Medda P, Bianchi C, Roncaglia I, Veltri A et al. Plasma Brain-Derived Neurotrophic Factor in treatment-resistant depressed patients receiving electroconvulsive therapy. Eur Neuropsychopharmacol 2009, 19:349-355, doi: 10.1016/j.euroneuro.2009. 01.002

63. Wilkinson ST, Kiselycznyk C, Banasr M, Webler RD, Haile C, Mathew $\mathrm{SJ}$ et al. Serum and plasma brain-derived neurotrophic factor and response in a randomized controlled trial of riluzole for treatment resistant depression. J Affect Disord 2018, 241:514-518, doi: 10.1016/j. jad.2018.08.075

64. Cheng CM, Hong CJ, Lin HC, Chu PJ, Chen MH, Tu PC et al. Predictive roles of brain-derived neurotrophic factor Val66Met polymorphism on antidepressant efficacy of different forms of prefrontal brain stimulation monotherapy: A randomized, double-blind, sham-controlled study. J Affect Disord 2021, 297:353-359, doi: 10.1016/j.jad. 2021.10.077

65. Haile CN, Murrough JW, losifescu DV, Chang LC, Al Jurdi RK, Foulkes A et al. Plasma brain derived neurotrophic factor (BDNF) and response to ketamine in treatment-resistant depression. Int J Neuropsychopharmacol 2014, 17:331-336, doi: 10.1017/S1461145713001119

66. Maffioletti E, Gennarelli M, Gainelli G, Bocchio-Chiavetto L, Bortolomasi M, Minelli A. BDNF Genotype and Baseline Serum Levels in Relation to Electroconvulsive Therapy Effectiveness in Treatment-Resistant Depressed Patients. J ECT 2019, 35:189-194, doi: 10.1097/YCT.000000 0000000583

67. Huang XB, Huang X, He HB, Mei F, Sun B, Zhou SM et al. BDNF and the Antidepressant Effects of Ketamine and Propofol in Electroconvulsive Therapy: A Preliminary Study. Neuropsychiatr Dis Treat 2020, 16:901908, doi: 10.2147/NDT.S248190

68. Allen AP, Naughton M, Dowling J, Walsh A, Ismail F, Shorten G et al. Serum BDNF as a peripheral biomarker of treatment-resistant depression and the rapid antidepressant response: A comparison of ketamine and ECT. J Affect Disord 2015, 186:306-311, doi: 10.1016/j. jad.2015.06.033

69. Pisoni A, Strawbridge R, Hodsoll J, Powell TR, Breen G, Hatch S et al. Growth Factor Proteins and Treatment-Resistant Depression: A Place on the Path to Precision. Front Psychiatry 2018, 9:386, doi:10.3389/ fpsyt. 2018.00386

70. Fukuda AM, Hindley LE, Kang JWD, Tirrell E, Tyrka AR, Ayala A et al. Peripheral vascular endothelial growth factor changes after transcranial magnetic stimulation in treatment-resistant depression. Neuroreport 2020, 31:1121-1127, doi: 10.1097/WNR.0000000000001523

71. Chávez-Castillo M, Núñez V, Nava M, Ortega Á, Rojas M, Bermúdez V et al. Depression as a Neuroendocrine Disorder: Emerging Neuropsycho- pharmacological Approaches beyond Monoamines. Adv Pharmacol Sci 2019, 2019:7943481, doi: 10.1155/2019/7943481

72. Pariante CM, Lightman SL. The HPA axis in major depression: classical theories and new developments. Trends Neurosci 2008, 31:464-468, doi: 10.1016/j.tins.2008.06.006

73. Stetler C, Miller GE. Depression and hypothalamic-pituitary-adrenal activation: a quantitative summary of four decades of research. Psychosom Med 2011, 73:114-126, doi: 10.1097/PSY.0b013e31820ad12b

74. Markopoulou K, Papadopoulos A, Juruena MF, Poon L, Pariante CM, Cleare AJ. The ratio of cortisol/DHEA in treatment resistant depression. Psychoneuroendocrinology 2009, 34:19-26, doi: 10.1016/j.psyneuen.2008.08.004

75. Dinan TG, Lavelle E, Cooney J, BurnettF, Scott L, Dash A. Dexamethasone augmentation in treatment-resistant depression. Acta Psychiatr Scand 1997, 95:58-61, doi: 10.1111/j.1600-0447.1997.tb00374.x

76. Kurczewska E, Ferensztajn-Rochowiak E, Jasinska-Mikolajczyk A, Chlopocka-Wozniak M, Rybakowski JK. Augmentation of Pharmacotherapy by Sleep Deprivation with Sleep Phase Advance in Treatment-Resistant Depression. Pharmacopsychiatry 2019, 52:186192, doi: 10.1055/a-0695-9138

77. Machado-Vieira R, Gold PW, Luckenbaugh DA, Ballard ED, Richards $E M$, Henter ID et al. The role of adipokines in the rapid antidepressant effects of ketamine. Mol Psychiatry 2017, 22:127-133, doi: 10.1038/ $\mathrm{mp} .2016 .36$

78. Bekhbat M, Chu K, Le NA, Woolwine BJ, Haroon E, Miller AH et al. Glucose and lipid-related biomarkers and the antidepressant response to infliximab in patients with treatment-resistant depression. Psychoneuroendocrinology 2018, 98:222-229, doi: 10.1016/j.psyneuen.2018.09.004

79. Papakostas GI, Petersen T, Sonawalla SB, Merens W, losifescu DV, Alpert JE et al. Serum cholesterol in treatment-resistant depression. Neuropsychobiology 2003, 47:146-151, doi: 10.1159/000070584

80. Veldman ER, Mamula D, Jiang H, Tiger M, Ekman CJ, Lundberg J et al. P11 (S100A10) as a potential predictor of ketamine response in patients with SSRI-resistant depression. J Affect Disord 2021, 290:240244, doi: 10.1016/j.jad.2021.04.055

81. Moaddel R, Luckenbaugh DA, Xie $Y$, Villaseñor $A$, Brutsche $N E$, Machado-Vieira $R$ et al. D-serine plasma concentration is a potential biomarker of $(\mathrm{R}, \mathrm{S})$-ketamine antidepressant response in subjects with treatment-resistant depression. Psychopharmacology (Berl) 2015, 232:399-409, doi: 10.1007/s00213-014-3669-0

82. Bhatt S, Nagappa AN, Patil CR. Role of oxidative stress in depression. Drug Discov Today 2020, 25:1270-1276, doi: 10.1016/j.drudis.2020. 05.001

83. Stirton $\mathrm{H}$, Meek BP, Edel AL, Solati $\mathrm{Z}$, Surendran A, Aukema $\mathrm{H}$ et al. Oxolipidomics profile in major depressive disorder: Comparing remitters and non-remitters to repetitive transcranial magnetic stimulation treatment. PLoS One 2021, 16:e0246592, doi: 10.1371/journal. pone. 0246592

84. Schmidt HD, Shelton RC, Duman RS. Functional biomarkers of depression: diagnosis, treatment, and pathophysiology. Neuropsychopharmacology 2011, 36:2375-2394, doi: 10.1038/npp.2011.151

85. Vinberg M, Weikop P, Olsen NV, Kessing LV, Miskowiak K. Effect of recombinant erythropoietin on inflammatory markers in patients with affective disorders: A randomised controlled study. Brain Behav Immun 2016, 57:53-57, doi: 10.1016/j.bbi.2016.05.006 


\title{
Peripheral prognostic biomarkers of response in treatment-resistant depression
}

\author{
Agorastos Agorastos, ${ }^{1-3}$ Theano Gkesoglou, ${ }^{1}$ Anastasios Kleidonopoulos, ${ }^{1}$ \\ Vasilios P. Bozikas ${ }^{1}$ \\ 'Second Department of Psychiatry, School of Medicine, Faculty of Health Sciences, Aristotle University of Thessaloniki, Greece, \\ ${ }^{2}$ VA Center of Excellence for Stress and Mental Health (CESAMH), VA San Diego Healthcare System, La Jolla, San Diego, CA, USA, \\ ${ }^{3}$ Institute of Agri-Food and Life Sciences Agro-Health, Hellenic Mediterranean University, Iraklion, Crete, \\ ${ }^{4}$ Second Department of Psychiatry, Psychiatric Hospital of Thessaloniki, Thessaloniki, Greece
}

\begin{abstract}
Major depressive disorder is a serious mental health disorder of high prevalence and the leading cause of disability worldwide. While there are several classes of therapeutic agents with proven antidepressant efficacy, only about $40-60 \%$ of patients respond to initial antidepressant monotherapy, and $30-40 \%$ of patients may even show resistance to treatment even under optimal antidepressant pharmacotherapy. Despite the existence of international guidelines, there are still no clear and widely accepted treatment algorithms, no established predictive biomarkers of response to treatment, while the management treatment-resistant depression is usually based on clinical experience. The present article offers a brief narrative review of studies published so far on the predictive quality of various blood-based peripheral biomarkers with respect to response to pharmacological, stimulation or behavioral treatment in patients with treatment-resistant depression. To summarize the results, there does not yet appear to be any specific biomarker that has sufficient discriminative predictive validity and can be used in the routine clinical practice of treating resistant depression. Many factors are likely to account for the above-mentioned research findings, including the wide variety of treatment protocols and the non-uniformly accepted definition of resistant depression used by the various studies, the small number of patients with treatment-resistant depression included, and the existence of different pathophysiological phenotypes of the disorder. The ineffective treatment of major depressive disorder requires an immediate improvement of our therapeutic approach by establishing clinically useful and easily accessible predictive biomarkers of response with high accuracy. The discovery of new and better clinical characterization of known biomarkers in the treatment of treatment-resistant depression could support a better staging and classification of the disorder, the development of personalized treatment algorithms for specific patient subgroups, the achievement of higher rates of stable remission, and the development of new precision drugs with minimal side effects.
\end{abstract}

KEYWORDS: Major depressive disorder, treatment-resistant depression, antidepressants, biomarkers, treatment response. 